\title{
The Mathematics of Non-Individuality
}

\author{
Décio Krause* \\ Department of Philosophy \\ Federal University of Santa Catarina \\ dkrause@cfh.ufsc.br
}

\begin{abstract}
"The development of the foundations of physics in the twentieth century has taught us a serious lesson. Creating and understanding these foundations turned out to have very little to do with the epistemological abstractions which were of such importance to the twentieth century critics of the foundations of mathematics: finiteness, consistency, constructibility, and, in general, the Cartesian notion of intuitive clarity. Instead, completely unforeseen principles moved into the spotlight: complementarity, and the nonclassical, probabilistic truth function. The electron is infinite, capricious, and free, and does not at all share our love for algorithms."
\end{abstract}

Yu. I. Manin, A course in mathematical logic, Springer, 1977, pp. 82-3.

\section{Introduction}

During the International Congress of Mathematicians, held in Paris in 1900, the great mathematician David Hilbert presented a list of 23 Problems of Mathematics which in his opinion should occupy the efforts of mathematicians in the century to come. To solve one of the problems become a way of achieving something really important in mathematics, and several Fields medals were awarded for this kind of endeavour. The sixth problem of his celebrated list dealt with the axiomatization of the theories of physics; Hilbert proposed "to treat in the same manner [as Hilbert himself had done with geometry], by means of axioms, those physical sciences in which mathematics plays an important part". In the XXth century, much was done in this direction, in continuation of those efforts already developed in the previous period, as remarked by Hilbert himself in his mentioned paper. In 1974, the American Mathematical Society sponsored a meeting to evaluate and to explore the consequences of Hilbert Problems. One

\footnotetext{
*Partially supported by CNPq.
} 
of the interesting implications of the Congress was that a new list of Mathematical Problems was proposed. According to Felix Browder, the editor of the Proceedings, this list was initiated by Jean Dieudonne through correspondence with mathematicians throughout the world.

The first problem of this new list deals with the foundations of mathematics, and was stated by the mathematician Yuri I. Manin; at item (b) (cf. quotation below), he makes reference to the needs of questioning the paradigm of classical set theory (the 'Cantor's paradise' referred to in the statement of the problem) on the basis of the treatment of collections of indistinguishable elementary particles in quantum mechanics, which (as he suggests) cannot be considered as standard 'sets'. It was then suggested that a 'new language' should be developed for such a purpose; let us see how Manin states his problem:

"In accordance with Hilbert's prophecy, we are living in Cantor's paradise. So we are bound to be tempted.

"Most mathematicians nowdays do not see any point in banning infinity, nonconstructivity, etc. Gödel made clear that it takes an infinity of new ideas to understand all about integers only. Hence we need a creative approach to creative thinking, not just a critical one. Two lines of research are naturally suggested.

"(a) to find out new axioms of (more or less naive) set theory, demonstrably efficient in number theory. Most advanced new methods ( $l$ adic cohomology) should be explored thoroughly. Are they readily formalized in Zermelo-Fraenkel or Gödel-Bernays systems ? Can we use in necessary categorical constructions only known axioms, or has something new already slipped in ?

"(b) We should consider possibilities of developing a totally new language to speak about infinity. Classical critics of Cantor (Brouwer et al.) argued that, say, the general choice axiom is an illicit extrapolation of the finite case.

"I would like to point out that this is rather an extrapolation of common-place physics, where we can distinguish things, count them, put them in some order, etc. New quantum physics has shown us models of entities with quite different behaviour. Even 'sets' of photons in a looking-glass box, or of electrons in a nickel piece are much less Cantorian than the 'set' of grains of sand. In general, a highly probabilistic 'physical infinity' looks considerably more complicated and interesting than a plain infinity of 'things'.

"Certainly there are no a priori reasons to choose fundamental concepts of mathematics so as to make them parallel to those of physics. Nevertheless it happened constantly and proved extremely fruitful. 
"The twentieth century return to Middle Age scholastics taught us a lot about formalisms. Probably it is time to look outside again. Meaning is what really matters."

When Manin says that "[w]e should consider possibilities of developing a totally new language to speak about infinity", he is obviously talking about set theory, also known as 'the theory of the infinite'. One of the reasons for doing this would be, as it is clear in the quotation, that collections of quantum objects would not be sets in the usual sense (cf. below). But why standard set theories like Zermelo-Fraenkel, von Neumann-Bernays-Gödel or Kelley-Morse are not appropriate (from a philosophical point of view) do deal with collections of quantum objects? (this was also proposed by other authors, as we shall see in the Introduction). And, if this is so, why physics still base itself in such mathematical frameworks with so great success? These are of course interesting questions related to the problem posed by Manin. In our opinion, the first question is linked to the way these mentioned theories (and classical logic in general) encompass a theory of identity: essentially, they impose to all mathematics they ground a Leibnizian structure, where there can be no indiscernible objects which are not the very same object (some form of Leibniz's Principle of the Identity of Indiscernibles, saying that there can be no 'two' objects sharing all their properties, is a theorem of standard mathematics). The second question, not considered here, ${ }^{1}$ which asks for the reason why we can deal with indistinguishable objects within 'classical' frameworks, can be explained in considering the use (even implicit) of some postulates we introduce in our physical theories, which can be summed up in the form of some Symmetrization Postulate. In short, quantum physics always encompass some principle of symmetry, imposing that permutations of indistinguishable objects are not to be regarded as observable, having no physical significance, as for instance when we take as 'significative' only symmetric and anti-symmetric vectors in a suitable Hilbert space. ${ }^{2}$ The use of such symmetry principles are in a sense equivalent of working within certain structures built in these set theoretical frameworks; so, although indiscernible within the structure, for instance being invariant by the automorphisms of the structure (as in the case of the Urelemente), the considered objects are distinguishable from the outside of the structure, namely, in the whole set-theoretical (well-founded) universe $\langle V, \in\rangle$, where the singletons can always be performed, and hence there exists always a property, namely, of being identical with $a$ (that is, to belong to $\{a\}$ ), which is characteristic of only $a$, for whatever $a$. But here we shall be concerned with the first question, that is, in presenting a mathematical framework which enable us to deal with indistinguishable objects taking as such from the start, that is, as a primitive idea. A further work would of course to show how to reconstruct quantum physics within such a framework; a first step in this direction is suggested en passant

\footnotetext{
${ }^{1}$ But see Krause \& Coelho 2002.

${ }^{2}$ For an account on these principles, see French \& Rickles 2002.
} 
here with the case of quantum statistics. Some works pointing other facts were have also been done already (see our references), but they will be not recalled here. We guess that, in realising such a project, that is, in expressing quantum theory in a mathematical framework like that one to be presented below, we will be carrying out quantum physics with an ontology of truly indistinguishable objects, contrary to what happens in the standard formulations, where, for instance, it is highly questioned whether there is a well defined ontological interpretation for the relevant vectors in, say, Hilbert spaces (cf. Bitbol 1996, Chap. 5).

The needs for such a 'new language' of 'sets' can perhaps be reinforced by Manin's own claim that "quantum mechanics does not really have its own language [but ] uses a certain fragment of the language of functional analysis". Manin's suggestions constitute a different way of saying that quantum theory is demanding for a different kind of logic. That is, in trying to answer his claims we are approaching 'quantum logic' from a radically different way than the usual study of the lattices associated to quantum theory, which become the standard approach ever since the pioneering work by von Neumann and Birkhoff in the $1930 \mathrm{~s}^{3}$ This way, we are looknig to a 'logic of quantum mechanics' as distinct from standard 'quantum logics'. In this paper, we introduce a mathematical formalism (termed Quasi Set Theory) which we think answers Manin's Problem and may contribute to express 'quantum facts' closer to intuition.

\section{Motivation}

In the late seventies, M. L. Dalla Chiara and G. Toraldo di Francia presented a quaset theory to provide the mathematical tools for the semantical analysis of the languages of microphysics. ${ }^{4}$ According to them, standard set theories are not adequate to represent microphysical phenomena, since the ontology of quantum physics apparently does not reduce to that of usual sets. One of the basic motivations underlying such a supposition is that collections of objects like elementary particles do not obey the axioms of set theories like Zermelo-Fraenkel due to the indistinguishability of their elements, since sets, as characterized by the axioms of standard set theories, stand for collections of individuals, which are distinguishable objects. In addition, they have suggested that identity questions from quantum theories demand a kind of intensional semantics, for which quaset theory should provide an adequate (meta)mathematical framework.

Starting from a distinct but related motivation, N. C. A. da Costa discussed the possibility of presenting logical systems in which some form of the Principle of Identity could be restricted. His motivations were essentially philosophical, in trying to show that the laws of classical logic are not so secure that they cannot be violated. ${ }^{5}$ Based on Schrödinger's ideas concerning the fact that the

\footnotetext{
${ }^{3}$ Concerning these approaches, see Dalla Chiara \& Giuntini 2001.

${ }^{4}$ Dalla Chiara \& Toraldo di Francia 1993; see also Dalla Chiara, Giuntini \& Krause 1998. Further details will be presented at section 5 below.

${ }^{5}$ da Costa 1980, pp. 117-120.
} 
concept of identity, or sameness, should lack sense with respect to elementary particles, ${ }^{6}$ da Costa introduced a two-sorted first order logic in which identity statements of the form $a=b$ make sense only with respect to the objects of one of the considered sorts; to the others (which should be regarded as denoting elementary particles), expressions like $x=y$ simply are not formulas. Hence, for these latter objects, it is not possible to say either that they are identical or that they are distinct from one another. ${ }^{7}$

Da Costa realized that a complete semantics could be stated for these 'Schrödinger Logics', but he noted that such a semantics, if grounded in the standard set theories, would not be adequate to express the intuitive idea of collections of objects for which the concept of identity lacks sense. Then he proposed that a kind of theory of quasi sets should be developed, in which standard sets were to be viewed as particular cases, and then, it was suggested, in such a theory a more adequate semantics for his logics would be achieved, although da Costa has not provided the details of such a quasi-set theory.

In 1990, a quasi set theory in this sense was proposed, ${ }^{8}$ and subsequently it has been improved in certain respects. ${ }^{9}$ The main motivation was not only to obtain a mathematical framework to provide semantics for Schrödinger logics, but also to pursue Schrödinger's intuitions and to explore the mathematical counterpart of a theory which admits collections of objects for which identity and diversity are meaningless concepts. But this should be done in such a way that, taking into account the motivation provided by the quantum mechanical treatment of elementary particles, a weaker concept of 'indistinguishability' could be considered as holding among certain elements.

In addition to Manin's problem mentioned in the Preface and in Dalla Chiara and Toraldo di Francia's above arguments, the importance of the development of such a mathematical framework may be viewed also from the following perspective. Ever since the 50's, Patrick Suppes has promulgated the idea that "to axiomatize a theory is to define a set theoretical predicate". ${ }^{10}$ This summarizes the fact that practically every concept of standard mathematics or even of empirical sciences, like physics, can be formulated (or expressed) within the scope of set theory. But, ever since T. Skolem in the $1920 \mathrm{~s}$, set theory has been axiomatized as a first order theory. ${ }^{11}$ So, loosely speaking, we may say that the axioms of a (either mathematical or physical) theory $T$ may be divided into three levels: (1) the "logical" ones (say, classical first order logic with or without equality), (2) the "mathematical" ones (say, the Zermelo-Fraenkel set theory) and (3) the specific axioms of the theory (for instance, axioms for groups, vector spaces, particle mechanics or quantum mechanics). Generally, when one speaks of the 'axioms of $T$ ', he means those of the third level, the other two becoming

\footnotetext{
${ }^{6}$ Schrödinger 1952, pp. 17-18.

${ }^{7}$ da Costa's system were extended to higher-order logics in da Costa and Krause 1994; 1997, following our previous work Krause 1990.

${ }^{8}$ See Krause 1990, 1992. That time, Dalla Chiara and Toraldo di Francia's quaset theory was still unknown to me.

${ }^{9}$ See Krause 1996, Dalla Chiara, Giuntini and Krause 1998, Sartorelli et al. 2001.

${ }^{10}$ Suppes 1957, 1967, 2002.

${ }^{11}$ Skolem 1922.
} 
implicit.

Of course that there are alternatives to the above schema, for instance by considering some higher order logic, category theory or a set theory other than Zermelo-Fraenkel to fulfill the axioms (1) and (2). We shall speak in general settheoretical terms in what follows in order to reinforce our point, by supposing that what is to be said can be adapted to these other alternatives as well. So, sometimes we speak in second-order languages, but the context will clean possible confusions.

If the axioms of levels (1) and (2) are taken to be those of 'classical' logic and set theory, and by this we may mean the classical first order logic (with or without identity) and a set theory like Zermelo-Fraenkel (ZF) (von NeumannBernays-Gödel (NGB) or Kelley-Morse (KM theories could be used instead) or even some other among the most common and used systems, then whatever theory $T$ based on this axiomatic basis is compromised with the Traditional Theory of Identity, that is, with the concept of identity as it is dealt with within such a logical/mathematical framework. As it is well known, despite some differences depending on the level of the employed language (either of first order or higher-order etc.), the concept of identity is captured by Leibniz's Law, which intuitively says that 'two' things are identical if and only if they share all their properties; in a second order language, we may write it as $x=y \operatorname{iff} \forall F(F(x) \leftrightarrow F(y))$ (here, identical things are of course the very same thing). ${ }^{12}$ The (extensional) set theoretical version asserts something similar, saying, by definition, that two things (either sets or the Urelemente) are identical iff they belong to the same sets, and that sets are identical iff they have the same elements (this is the Axiom of Extensionality, AE). In particular, it can be proven that Leibniz' Principle of the Identity of the Indiscernibles (PII for short), in symbols, $\forall F(F(x) \leftrightarrow F(y) \rightarrow x=y$, is a theorem of higher order logics (equivalently, $\forall z(x \in z \leftrightarrow y \in z) \rightarrow x=y$ is a trivial theorem of the theories of sets just mentioned, due to AE). Hence, classical logic and mathematics in a certain sense vindicate Leibniz's dictum that there are no two entities which differ solo numero, and this should be so for whatever theory whose axioms (1) and (2) are 'classical.

Notwithstanding, several authors have questioned the validity of PII in quantum theory, mainly due to the existence of absolutely indistinguishable quanta, which share all their attributes (or properties) and, even so, are of course not the same object. ${ }^{13}$ So, it seems clear that if we agree with quantum theory in considering indistinguishable but not identical quanta, then there is an interesting foundational problem involved in this discussion, for collections of objects which are indistinguishable contradict Cantor's 'definition' of the concept of set, namely, "by an aggregate (Menge) we are to understand any collection into a whole $M$ of definite and separate objects $m$ of our intuition or our thought" ,${ }^{14}$ an intuitive concept which is maintained within the axiomatic framework of standard (extensional) set theories. By the above, this problem, namely, that of

\footnotetext{
${ }^{12}$ For details on the traditional theory of identity, see Krause and Coelho 2002.

${ }^{13}$ Cf. Dalla Chiara and Toraldo di Francia 1995.

${ }^{14}$ Cantor 1955, p. 85.
} 
finding axioms for dealing with collections of (truly) indistinguishable objects, can be termed 'the Manin Problem'.

Quasi-set theory is a way of considering collections of indistinguishable but not identical objects. The theory has been proposed precisely to provide a mathematical tool for describing collections of objects whose indistinguishability is considered right at the start, as demanded by Heinz Post in regarding quantum objects. ${ }^{15}$ Generally, the ways of dealing with this concept within standard mathematics tend to 'mask' the distinguishability of the elements of a set (in the Cantorian sense just recalled) by 'passing the quotient' by some equivalence relation and saying that those elements that belong to the same equivalence class are 'indistinguishable' (by the way, this is what did Hermann Weyl in his treatment of the concept of 'aggregate of individuals'). ${ }^{16}$ Alternatively, we could use groups of (permutation) symmetries in such a way that particles of the same sort (but different as individuals) are treated as 'identical'. ${ }^{17}$ But these approaches, typical of the standard developments of quantum theory, grounded on the idea of invariance by automorphisms, groups and the like, are artificial in the sense that the considered objects (individuals) are firstly taken as belonging to a set (hence being distinguishable entities) and then their individuality is effectively rendered into a 'mock' form by some mathematical device. This kind of trick makes physics works, but the philosophically more interesting problem would be to look for a mathematical framework by means of which certain elements (denoting elementary particles) could be supposed indistinguishable from the beginning. This is what quasi-set theory aims to do.

\section{The Quasi-Set Theory $\mathfrak{Q}$}

The quasi set theory we shall present here presents some modifications from earlier versions, ${ }^{18}$ and encompass more details and explanations. We call $\mathfrak{Q}$ such a theory, which is based on ZFU-like axioms (Zermelo-Fraenkel with Urelemente) and having the classical first order predicate calculus without identity as its underlying logic. However, instead of just one kind of atoms, the theory allows the existence of two sorts of Urelemente, termed $m$-atoms and $M$-atoms (two primitive unary predicates express that: $m(x)$ says that $x$ is an $m$-atom and $M(x)$ says that $x$ is an $M$-atom). There are still the binary primitive predicates $\equiv$ (indistinguishability) and $\in$ (membership), one unary functional symbol $q c$ (quasi-cardinal) and a unary predicate letter $Z(Z(x)$ says that $x$ is a set, and these will correspond to the sets of ZFU). Of course quasi-set theory could be formulated as a higher-order theory, or even be based on other kind of axioms, like $N B G$, perhaps with some advantages but such an approach will be not considered here (although something in this sense is suggested below).

\footnotetext{
${ }^{15}$ Post 1963.

${ }^{16}$ Weyl 1949, App. B.

${ }^{17}$ See the paper by E. Castellani in Castellani 1998.

${ }^{18}$ Krause 1996; Dalla Chiara, M. L., Giuntini, R. and Krause, D. 1998, being closer to Sartorelli et al. 2001.
} 
The basic idea is that the $M$-atoms have the properties of standard Urelemente of ZFU, while the $m$-atoms may be thought of as representing the elementary particles of quantum physics. ${ }^{19}$ With regard to this last kind of entities, the concept of identity should make no sense. ${ }^{20}$ In quasi set theory, this restriction is achieved by restricting the concept of formula: expressions like $x=y$ are not well formed if $x$ and $y$ denote $m$-atoms. The equality symbol is not primitive, but a concept of extensional identity (represented by ${ }_{E}$ ) is defined so that it has all the properties of standard identity of ZFU. Then, the axiomatics permits us to differenciate between the concepts of (extensional) identity (being the very same object) and indistinguishability (agreement with respect to all the attributes).

A quasi-set (qset for short) $x$ is defined as something which is not an Urelement. A qset $x$ may have a cardinal (termed its quasi cardinal, and denoted by $q c(x)$ ), but the idea is that the theory does not associate to certain qsets an ordinal, since there should be quasi sets which cannot be ordered (since their elements are to be indistinguishable $m$-atoms, expressed by the relation $\equiv)$. The concept of quasi cardinal is then taken as primitive, since (in principle) it cannot be defined by usual means (that is, as particular ordinals). ${ }^{21}$ This fits the idea that quantum particles cannot be ordered or counted, but only aggregated in certain amounts. Notwithstanding, due to the concept of quasi cardinal, there is a sense in saying that there may exist a certain quantity of $m$-atoms obeying certain conditions, although they cannot be named or labelled. (Below we shall also present a variant of the theory $\mathfrak{Q}$ where the quasi-cardinal of a qset $x$ may vary in time - see section 6 , so as a theory $\mathfrak{Q}^{m}$ which explicit postulate the existence of $m$-atoms.)

So, let us begin by describing the formal details of the theory $\mathfrak{Q}$. From now on, we sometimes shall use relativised quantifiers as follows: $\forall_{P} x \alpha(x)$ means $\forall x(P(x) \rightarrow \alpha(x))$, while $\exists_{P} x \alpha(x)$ stands for $\exists x(P(x) \wedge \alpha(x))$, where $P$ is a suitable predicate.

\section{Definition 1}

(i) [Quasi-set (qset)] $Q(x)=_{\mathrm{df}} \neg(m(x) \vee M(x))$

(ii) [Pure qset] (a collection of indistinguishable $m$-atoms) $P(x)={ }_{\mathrm{df}} Q(x) \wedge$ $\forall y(y \in x \rightarrow m(y)) \wedge \forall y \forall z(y \in x \wedge z \in x \rightarrow y \equiv z)$

(iii) [Dinge] (either 'sets' or the Urelemente) $D(x)={ }_{\mathrm{df}} M(x) \vee Z(x)$ (these are the '(classical) things', to use Zermelo's original terminology). ${ }^{22}$

\footnotetext{
${ }^{19}$ Of course this can be generalised to cope with more recent advances in physics, like collections of strings and branes, but we shall keep the terminology as usual in these contexts. By the way, Stephen Hawking says that "all the branes are created equal" -Hawking 2001, p. 54 , which suggests that a collection of them should be taken as a quasi-set.

${ }^{20}$ This idea follows Schrödinger 1952, pp. 17-18, but the same can be said for instance of strings of String Theory (or of branes): in what sense can we say that two strings are distinct? Apparently, as in the case of particles, Schrödinger's ideas apply also here. In a sense, string theory brings again an ontology of 'objects' of a sort, of course indistinguishable ones.

${ }^{21}$ But see the remarks made below at section 3.2.

${ }^{22}$ Zermelo 1908.
} 
(iv) [A qset whose elements are also qsets] $E(x)={ }_{\mathrm{df}} Q(x) \wedge \forall y(y \in x \rightarrow Q(y))$

(v) [Extensional Identity]

$$
\begin{aligned}
& x={ }_{E} y={ }_{\mathrm{df}}(Q(x) \wedge Q(y) \rightarrow \forall z(z \in x \leftrightarrow z \in y)) \vee(M(x) \wedge M(y) \\
&\left.\rightarrow \forall_{Q} z(x \in z \leftrightarrow y \in z)\right),
\end{aligned}
$$

where $\forall_{Q}$ is the universal quantifier relativized to qsets.

(vi) [Subqset] For all qsets $x$ and $y, x \subseteq y={ }_{\mathrm{df}} \forall z(z \in x \rightarrow z \in y)$

The first axioms of $\mathcal{Q}$ are the following:

(Q1) $\forall x(x \equiv x)$

(Q2) $\forall x \forall y(x \equiv y \rightarrow y \equiv x)$

(Q3) $\forall x \forall y \forall z(x \equiv y \wedge y \equiv z \rightarrow x \equiv z)$

(Q4) $\forall x \forall y\left(x={ }_{E} y \rightarrow(A(x, x) \rightarrow A(x, y))\right)$, with the usual syntactic restrictions, that is, $A(x, x)$ is a formula whatever and $A(x, y)$ arises from $A(x, x)$ by the substitution of some free occurrences of $x$ by $y$, provided that $y$ is free for $x$ in $A(x, x)$.

Theorem 2 Whether $Q(x)$ or $M(x)$, then $x={ }_{E} x$.

Proof: If $Q(x)$, since $\forall z(z \in x \leftrightarrow z \in x)$, then $x={ }_{E} x$ by the definition of extensional identity. If $M(x)$, then of course for all qset $z$, we have that $x \in z \leftrightarrow x \in z$, so $x={ }_{E} x$. I

(Q5) Nothing is at the same time an $m$-atom and an $M$-atom: $\forall x(\neg(m(x) \wedge$ $M(x)))$.

Theorem 3 Whether $Q(x)$ or $M(x)$, then $\neg m(x)$.

Proof: If $Q(x)$, then $\neg m(x)$ by the definition of qset. If $M(x)$, then $\neg m(x)$ by Q5. I

(Q6) The atoms are empty: $\forall x \forall y(x \in y \rightarrow Q(y))$.

In the next sections we shall discuss a little bit on the existence of atoms. This last axiom is interesting from the perspective of physics, for it seems that we should suppose that the $M$-atoms could be 'composed' of $m$-atoms in some way. This is precisely what should be given the physical interpretation we have suggested, but in this case the relationship between the atoms would be not membership. In this case, what seems to be required is some form of mereologic theory suitable for expressing this relationship, but such a theory has yet to be constructed. ${ }^{23}$

\footnotetext{
${ }^{23}$ Concerning mereology, or "the theory of part-whole", first developed by the Polish logician S. Lesniewski, see Simons 1987. The idea is to add to que axioms of the theory $\mathfrak{Q}$ suitable mereological axioms.
} 
(Q7) Every set is a qset: $\forall x(Z(x) \rightarrow Q(x))$.

(Q8) Qsets whose elements are 'classical things' are sets and conversely:

$$
\forall_{Q} x(\forall y(y \in x \rightarrow D(y)) \leftrightarrow Z(x))
$$

Our intention is to characterize sets in $\mathfrak{Q}$ so that they can be identified with the standard sets of ZFU. This should be achieved if they were taken to be those qsets whose transitive closure ${ }^{24}$ do not contain $m$-atoms. The ' $\rightarrow$-part' of Q8 gives half of the answer: if all the elements of $x$ are Dinge (sets of $M$-atoms), then $x$ is a set. Concerning the converse, it is not enough to postulate that no element of a set is an $m$-atom, since it may be that the elements of its elements have $m$-atoms as elements and so on. But this question can be rightly answered if we have $Z(x) \rightarrow \forall y(y \in x \rightarrow D(y))$, which is precisely the ' $\leftarrow$-part' of Q8.

(Q9) This axiom is the conjunction of the following three sentences:

$$
\begin{aligned}
& \forall x \forall y(m(x) \wedge x \equiv y \rightarrow m(y)) \\
& \forall x \forall y\left(x==_{E} y \wedge M(x) \rightarrow M(y)\right) \\
& \forall x \forall y\left(x={ }_{E} y \wedge Z(x) \rightarrow Z(y)\right)
\end{aligned}
$$

(Q10) [The empty qset] There exists a qset (denoted by ' $\emptyset$ ') which does not have elements: $\exists_{Q} x \forall y(\neg(y \in x))$.

Theorem 4 The empty qset is a set.

Proof: $\quad$ Take $x={ }_{E} \emptyset$. Since $y \in x$ is false by Q10, then the antecedent of $\forall y(y \in x \rightarrow D(x))$ is true, hence $Z(\emptyset)$ by $\mathrm{Q} 8$.

(Q11) Indistinguishable Dinge ${ }^{25}$ (see Def. 1) are extensionally identical:

$$
\forall_{D} x \forall_{D} y\left(x \equiv y \rightarrow x={ }_{E} y\right) .
$$

Theorem 5 The relation of extensional equality has all the properties of classical equality.

Proof: Being $x$ such that $D(x)$ then $x \equiv x \rightarrow x={ }_{E} x$ by Q11; the axiom Q4 provides substitutivity for Dinge; so, the standard axioms for first-order identity are obtained.

Theorem 6 If $M(x)$ and $x \equiv y$, then $M(y)$; the same holds for 'sets', namely, $Z(x)$ and $x \equiv y$ entails $Z(y)$.

\footnotetext{
${ }^{24}$ This concept has its usual sense: $T C(x)=_{\mathrm{df}} x \cup \bigcup x \cup \bigcup \bigcup x \ldots$

${ }^{25}$ Zermelo's set theory is concerned with a domain (Bereich) of individuals, the sets and the Urelemente, which he referred to simply as objects (Dinge).
} 
Proof: (For $M$-atoms) Suppose that $M(x)$ and $x \equiv y$. If $m(y)$, since $y \equiv x$ by Q2, then $m(x)$ by Q9. So, $M(y)$ or $z(y)$. But, by Q11, being $x$ an $M$-atom, $x \equiv y$ entails $x={ }_{E} y$, hence by $\mathrm{Q} 4$, if $M(x)$ stands for $A(x, x)$, we get $M(y)$. Similar things happen if we suppose $Z(y)$.

Remark: The distinction between extensional identity and the primitive indistinguishability may be seen as follows, although the formal details can be provided only after other axioms being stated. By the above axioms and theorems, the indistinguishability relation $\equiv$ permits substitutivity for all primitive non logical symbols, except membership. That is, if $B$ is $m, M, Z$ or even $q c$, then $B(x) \wedge x \equiv y \rightarrow B(y)$ is a theorem. If this was possible also for $\in$, then since $\equiv$ is reflexive (Axiom Q1), we would have full substitutivity for $\equiv$, hence it could be not distinguished from usual identity. ${ }^{26}$ But concerning membership, this is not the case, that is, $x \in w \wedge y \equiv x$ does not entail that $y \in z$, for the theory has no axioms that grant this fact. So, indistinguishability is not 'standard' identity.

(Q12) [Weak-Pair] For all $x$ and $y$, there exists a qset whose elements are indistinguishable from either $x$ or $y: \forall x \forall y \exists_{Q} z \forall t(t \in z \leftrightarrow t \equiv x \vee t \equiv y)$.

We denote this qset by $[x, y]$. When $x$ and $y$ are Dinge, we may use the usual notation $\{x, y\}$. Let us remark that $[x, y]$ stand for the qset of the indistinguishable from either $x$ or $y$, and in general may contain more that two elements.

(Q13) [The Separation Schema] By considering the usual syntactical restrictions on the formula $A(t)$, that is, $A(t)$ being a well formed formula in which $t$ is free, the following is an axiom schema:

$$
\forall_{Q} x \exists_{Q} y \forall t(t \in y \leftrightarrow t \in x \wedge A(t))
$$

This qset is written $[t \in x: A(t)]$, and $\{t \in x: A(t)\}$ when such a qset is a set.

(Q14) [Union] $\forall_{Q} x\left(E(x) \rightarrow \exists_{Q} y(\forall z(z \in y) \leftrightarrow \exists t(z \in t \wedge t \in x))\right)$

This qset is denoted by $\bigcup x$ or by $\bigcup_{t \in x} t$ or even by $u \cup v$ when $t$ has just two elements (qsets) $u$ and $v$.

(Q15) [Power-qset] $\forall_{Q} x \exists_{Q} y \forall t(t \in y \leftrightarrow t \subseteq x)$.

According to the standard notation, we write $\mathcal{P}(x)$ for this qset.

\section{Definition 7}

(i) ['Ordered pair'] $\langle x, y\rangle=_{\mathrm{df}}[[x],[x, y]]$

\footnotetext{
${ }^{26}$ Shoenfield 1967, Chap. 1.
} 
(ii) [Weak Singleton] $[x]=[x, x]$ (this is the collection of the indistinguishable from $x$ )

(iii) $x \times y=_{\mathrm{df}}[\langle z, u\rangle \in \mathcal{P} \mathcal{P}(x \cup y): z \in x \wedge u \in y]$

As in the case of $[x, y],[x]$ is the qset of all those indistinguishable from $x$, so it may have more than one element. The same may be said for the cartesian product of two qsets etc. The concepts of intersection and difference of qsets are defined in the usual way so that $t \in x \cap y$ iff $t \in x \wedge t \in y$ and $t \in x-y$ iff $t \in x \wedge t \notin y$.

(Q16) [Infinity] $\exists_{Q} x(\emptyset \in x \wedge \forall y(y \in x \wedge Q(y) \rightarrow y \cup[y] \in x))$.

(Q17) [Regularity] (Qsets are well-founded):

$$
\forall_{Q} x\left(E(x) \wedge x \neq_{E} \emptyset \rightarrow \exists_{Q} y\left(y \in x \wedge y \cap x={ }_{E} \emptyset\right)\right) .
$$

Of course this axiom raises another cluster of questions, for if the $m$-atoms are to be thought of as representing elementary particles, then apparently we are faced with the old problem of continuously dividing up a certain object, and our axiom may suggest that we are proposing that such a 'division' should have an end. But of course this is not so, for the axiom talks in terms of qsets; every qset has a qset as element to which it has no element in common, but nothing is said about atoms. With regard to these ones, the problem regarding a mereology remains; at least in principle, we should agree with Heisenberg when he says that (in quantum physics) "the concept of 'dividing' has lost its meaning". ${ }^{27}$

\subsection{Relations and quasi-functions}

In this section we shall see that relations and functions cannot be defined in quasi-set theory as in standard mathematics. The basic motive is that functions cannot distinguish between arguments and values if there are $m$-atoms involved. Furthermore, due to the lack of sense of speaking in $\mathfrak{Q}$ about the identity and about the difference of $m$-atoms, ordering relations cannot be adequately defined on a qset with indistinguishable $m$-atoms as elements. We shall pay attention to binary relations only, but of course the considerations below can be generalized. Let us see the details.

Definition 8 A qset $w$ is a quasi-relation (we shall call them only 'relations') between $x$ and $y$ if it satisfies the following predicate $R$ :

$$
R(w)={ }_{\mathrm{df}} Q(w) \wedge \forall z\left(z \in w \rightarrow \exists u \exists v\left(u \in x \wedge v \in y \wedge z={ }_{E}\langle u, v\rangle\right)\right)
$$

As usual we sometimes write $u w v$ for $\langle u, v\rangle \in w$. Relations are important in general for characterizing attributes of the elements of certain collection of

${ }^{27}$ Heisenberg, 'The nature of elementary particles', reprinted in Castellani 1998. 
objects. In standard set theory, ordering relations, let us recall, are of two main basic types: partial orderings are those binary relations $P$ on a set $A$ which are (i) reflexive, that is, $\forall x(x P x)$, (ii) anti-symmetric, that is, $\forall x \forall y(x P y \wedge y P x \rightarrow$ $x=y$ ), and (iii) transitive, $\forall x \forall y \forall z(x P y \wedge y P z \rightarrow x P z)$. If $P$ is also (iv) connected, that is, $\forall x \forall y(x \neq y \rightarrow x P y \vee y P x)$, then it is a total (or linear) ordering. It is easy to see why such relations cannot be defined on a qset whose elements are indistinguishable $m$-atoms: without the relation of identity, we cannot even state the definition. But what about the so-called strict partial and total orderings?

Let us recall that a strict partial ordering on a set $A$ is a binary relation $S$ on $A$ such that (i) $S$ is irreflexive, that is, $\forall x \neg(x S x)$ and (ii) transitive. An strict total ordering on $A$ is one which is irreflexive, transitive and connected. The reader could rightly say that a binary relation $w$ on a qset $x$ (that is, obeying the predicate $R$ of the above definition) such that (a) $w$ is irreflexive, (b) transitive and (c) for every $u$ and $v$ in $x$ is such that $u w v \vee v w u$, should be regarded as a strict total ordering on $x$, although we cannot say that $u$ and $v$ are distinct. What are the consequences of this result?

We should recall that in considering sets, we always can (at least in principle) label whatever of is elements, say by associating their singletons to them: for instance, associate $\{x\}$ to $x$. In extensional contexts, this singleton can be viewed as a 'property' of $x$ only (remember that Leibniz Law holds in classical set theory, so the elements of a set are 'individuals' in a sense). But in $\mathfrak{Q}$ this cannot be done if $x$ is an $m$-atom, for the 'singleton' $[x]$ (as it results from the remaining axioms to be stated below) cannot be said to have cardinal 1 (in the case, we should say 'quasi-cardinal'). So, when we say that $u w v$, that is, $\langle u, v\rangle \in w$, we should remember the that by the definition of the 'ordered pair' given above, $\langle u, v\rangle \in w$ means $[[u],[u, v]]$ and being $u \equiv v$, this pair is indistinguishable (in the sense of the axiom of Weak Extensionality to be presented below) from $[[v],[v, u]]$, which is the 'ordered pair' $\langle v, u\rangle$. Furthermore, this qset is also indistinguishable from $[[u]]$, that is, from $\langle u, u\rangle$. Of course the theory does not imply that $\langle v, u\rangle$ (or that $\langle u, u\rangle$ ) also belongs to $w$, but the relation $w$ is indistinguishable (in the same sense) from the relations $w^{\prime}$ and $w^{\prime \prime}$, which have these pairs as elements (supposing that the other elements do not provide any further distinction between $w, w^{\prime}$ and $\left.w^{\prime \prime}\right)$.

This means that whatever strict total order $w$ on a qset $x$ of indistinguishable $m$-atoms such that $\langle u, v\rangle \in w$ is confused in the theory (they are indistinguishable) with another $w^{\prime}$ such that $\langle v, u\rangle \in w^{\prime}$ (the same holds with respects to $w^{\prime \prime}$ above), and no order could be said to make sense on $x$ within $\mathfrak{Q}$, for the theory cannot distinguish the defined order from another one that has its elements in a 'reverse' order (the reader should remind that the idea of a 'reverse order' requires identification of the elements).

So, ordering relations (on a pure qset whose elements are indistinguishable $m$-atoms) don't have detectable significance, for the theory doesn't distinguish between such relations from those which (intuitively speaking) have the 'same' elements in a reverse order. This point has a certain parallel in physics. Suppose that a certain atom releases an electron $u$ becoming an ion. Later, an electron 
$v$ is captured by the atom, which becomes neutral again. What is the difference between the original atom and the (again) neutral atom? Well, as we know, there are no differences, for quantum physics cannot distinguish between $u$ and $v$ (let us remark that, in our theory, a theorem below will give us a mathematical interpretation of this result). But there is a sense in saying that $u$ and $v$ are in certain 'order', for one electron was released while 'another' one was captured. But this 'ordering' is only meta-theoretical (the difference is only in mente Dei, as Dalla Chiara and Toraldo di Francia could say). The same can be said concerning the orderings $w, w^{\prime}$ and $w^{\prime \prime}$ above. Whatever distinction among then is only metamathematical and cannot the given within $\mathfrak{Q}$.

Definition 9 [Quasi-functions] If $x$ and $y$ are qsets and $R$ is the predicate for 'relation' defined above, we say that $f$ is a quasi-function (q-function) with domain $x$ and counter-domain $y$ if it satisfies the following predicate:

$$
\begin{aligned}
& Q F(f)=_{\mathrm{df}} R(f) \wedge \forall u(u \in x \rightarrow \exists v(v \in y \wedge\langle u, v\rangle \in f)) \wedge \\
& \forall u \forall u^{\prime} \forall v \forall v^{\prime}\left(\langle u, v\rangle \in f \wedge\left\langle u^{\prime}, v^{\prime}\right\rangle \in f \wedge u \equiv u^{\prime} \rightarrow v \equiv v^{\prime}\right)
\end{aligned}
$$

Furthermore, $f$ is a $q$-injection iff $f$ is a $q$-function from $x$ to $y$ and satisfies the additional condition:

$$
\begin{aligned}
\forall u \forall u^{\prime} \forall v \forall v^{\prime}(\langle u, v\rangle \in f \wedge & \left.\left\langle u^{\prime}, v^{\prime}\right\rangle \in f \wedge v \equiv v^{\prime} \rightarrow u \equiv u^{\prime}\right) \\
& \wedge q c(\operatorname{Dom}(f)) \leq_{E} q c(\operatorname{Rang}(f)) .
\end{aligned}
$$

In the same vein, $f$ is a $q$-surjection iff it is a function from $x$ to $y$ such that

$$
\forall v(v \in y \rightarrow \exists u(u \in x \wedge\langle u, v\rangle \in f)) \wedge q c(\operatorname{Dem}(f)) \geq_{E} q c(\operatorname{Ran} g(f)) .
$$

Finally, an $f$ which is both a $q$-injection and a $q$-surjection is said to be a $q$-bijection. In this case, $q c(\operatorname{Dom}(f))={ }_{E} q c(\operatorname{Rang}(f))$, where $\operatorname{Dom}(F)$ and $\operatorname{Rang}(F)$, respectively the domain and the range of $f$ have their usual meanings (but the reader should note that due to the lack of individuality of the $m$ atoms, these qsets have a peculiar characteristics, which is expressed by the Theorem of the Unobservability of Permutations mentioned below). As it is easy to see, when there are no $m$-atoms involved, the above concept coincides with the standard definition of a function.

To summarize, we can state the following theorem:

Theorem 10 Nor partial nor total ordering relations can be defined on a pure qset whose elements are indistinguishable from one another.

Proof : (Sketch) The definitions of partial and total orders require antisymmetry, and this property cannot be stated without identity. Asymmetry also cannot be supposed. In fact, if $x \equiv y$, then for every $R$ such that $\langle x, y\rangle \in R$, it follows that $\langle x, y\rangle={ }_{E}[[x]]={ }_{E}\langle y, x\rangle \in R$; so, $x R y$ entails $y R x$. 


\subsection{Quasi-Cardinals}

For presenting the remaining axioms, we need to realise that a 'copy' of ZFU can be defined within $\mathfrak{Q}$. Let us see the main idea. Firstly, we need to define the translation from the language of ZFU to the language of $\mathfrak{Q}$. This will show that the theory $\mathfrak{Q}$ encompasses a 'classical' counterpart which coincides with ZFU.

The translation can be defined in the following way: let $A$ be a formula of the language of ZFU (which we may admit has an unary predicate $S$ which stands for 'sets'). Then, call $A^{q}$ its translation to the language of $\mathfrak{Q}$, defined as follows:

(i) If $A$ is $S(x)$, then $A^{q}$ is $Z(x)$

(ii) If $A$ is $x=y$, then $A^{q}$ is $\left((M(x) \wedge M(y)) \vee(Z(y) \wedge Z(y)) \wedge x=_{E} y\right)$

(iii) If $A$ is $x \in y$, then $A^{q}$ is $((M(x) \vee Z(x)) \wedge Z(y)) \wedge x \in y$

(iv) If $A$ is $\neg B$, then $A^{q}$ is $\neg B^{q}$

(v) If $A$ is $B \vee C$, then $A^{q}$ is $B^{q} \vee C^{q}$

(vi) If $A$ is $\forall x B$, then $A^{q}$ is $\forall x(M(x) \vee Z(x) \rightarrow B)$

Theorem 11 If $A$ is an axiom of ZFU and $A^{q}$ is its translation to the language of $\mathfrak{Q}$ given by the above definition, then $A^{q}$ is a theorem of $\mathfrak{Q}$.

This theorem, whose proof can be given by careful checking, shows that if $\mathfrak{Q}$ is consistent, so is ZFU.

The above result shows that there is a copy of ZFU in $\mathcal{Q}$ (Figure 1). In this 'copy', we may define as usual the following concepts: $C d(x)$ for ' $x$ is a cardinal'; $\operatorname{card}(x)$ denotes 'the cardinal of $x$, and Fin $(x)$ says that ' $x$ is a finite quasi-set'. Then, by considering these concepts, we may present the axioms for quasi-cardinals:

(Q18) Every object which is not a qset (that is, every Urelement) has quasicardinal zero: $\forall x\left(\neg Q(x) \rightarrow q c(x)={ }_{E} 0\right)$.

(Q19) The quasi-cardinal of a qset is a cardinal (defined in the 'classical part' of the theory) and coincides with its cardinal itself when this qset is a set: ${ }^{28}$

$$
\forall_{Q} x \exists ! y\left(C d(y) \wedge y={ }_{E} q c(x) \wedge\left(Z(x) \rightarrow y={ }_{E} \operatorname{card}(x)\right)\right)
$$

So, we are postulating that whatever qset has a quasi-cardinal and that such quasi-cardinal is a cardinal (as defined by usual means in the 'standard' part of the theory). This axiom apparently is contrary to the result mentioned

\footnotetext{
${ }^{28}$ Really, 'sets in $\mathfrak{Q}$, that is, 'copies' of ZFU sets.
} 


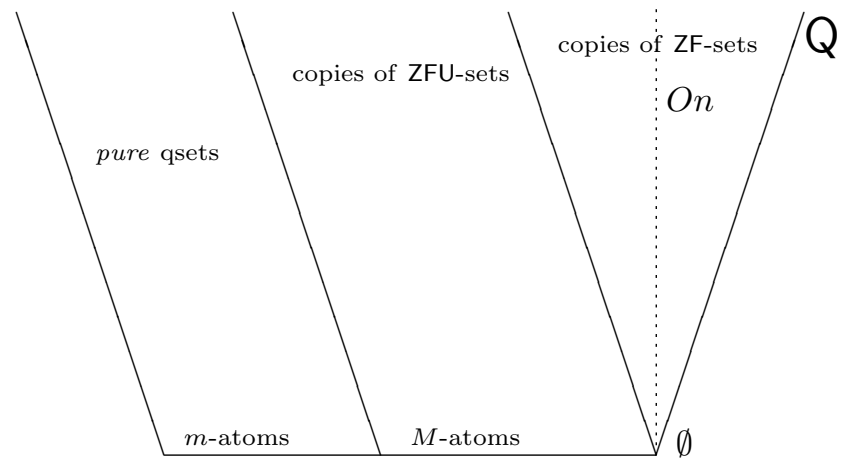

Figure 1: The Quasi-Set Universe

in the preceding section which shows that no order relation can be defined on a qset of indistinguishable $m$-atoms, for the existence of its quasi-cardinal seems to suggest that whatever quasi-set (including those whose elements are indistinguishable $m$-atoms) can be ordered, since, if defined as usual, a cardinal is a particular ordinal. The explanation of this fact is that the associated ordinal of a quasi-set of indistinguishable $m$-atoms cannot be something that belongs to the 'classical' part of the theory (in the figure above, it would lie in the region above $m$ of the 'pure' qsets). Alternatively, we could say, as in the case of the Skolem's paradox, that such an ordinal does not belong to the theory at all! (in the sense that its existence cannot be derived from the axioms). This is of course an interesting point to be further considered, which we do not pursue here. But what results is that perhaps for physical applications a different concept of cardinal should be used instead of the standard one, for at least in the cases involving quantum objects, it should be interesting to have a process of counting which does not induces any kind of order. For instance, perhaps we could take a definition in the sense of Frege-Russell (as a certain class of equinumerous classes). ${ }^{29}$ Another alternative could be achieved by Enderton's definition of the concept of $k c a r d ;{ }^{30}$ Enderton suggests that $k \operatorname{card}(x)$ is to be the set of all sets $y$ equinumerous to $x$ and having the least possible rank. Then, $k \operatorname{card}(x)=k \operatorname{card}(y)$ iff $x$ and $y$ are equinumerous, as desired, but the definition of $k c a r d$ relies on regularity, and not on the axiom of choice (which in

\footnotetext{
${ }^{29}$ More precisely, the cardinal of a set $X$ would be $\operatorname{card}(X)=_{\mathrm{df}}\{Y: Y \approx X\}$, where $\approx$ means 'equinumerous to' (Suppes 1960, p. 109). But the problems with such a definition are well known (op. cit.).

${ }^{30}$ We thank Antonio Coelho for recalling us this definition.
} 
the standard definition is used to show that every set has a cardinal number), ${ }^{31}$ so for a finite nonempty set $x, k \operatorname{card}(x)$ fails to be a natural number (which could be not so good to physics). Perhaps we could eliminate from the axiom above the fact that $q c(x)$ is a cardinal and leave its characterization to the particular model to be considered. Anyway, it seems clear that the search for a more adequate definition of cardinal which could be useful to physics is something to be investigated further.

Finally, still regarding the existence of quasi-cardinals, we remark that for the discussion on quantum field theory, ${ }^{32}$ one might guess that it would be interesting that in certain situations we couldn't say that the quantity of elements in a certain qset is fixed (the case of virtual particles comes to the mind). But it is not necessary to fix this fact here, for the possibility of varying the quasicardinal in time (as given in the theory $\mathfrak{Q}^{t}$ sketched below) and the concept of the cloud of a qset (also given below) shall provide the mathematical insights for the relevant discussion. Let us turn now to the other axioms.

(Q20) Every non-empty qset has a non null quasi-cardinal:

$$
\forall_{Q} x\left(x \neq_{E} \emptyset \rightarrow q c(x) \neq_{E} 0\right)
$$

(Q21) $\forall_{Q} x\left(q c(x)={ }_{E} \alpha \rightarrow \forall \beta\left(\beta \leq_{E} \alpha \rightarrow \exists_{Q} y\left(y \subseteq x \wedge q c(y)={ }_{E} \beta\right)\right)\right.$

(Q22) $\forall_{Q} x \forall_{Q} y\left(y \subseteq x \rightarrow q c(y) \leq_{E} q c(x)\right)$

(Q23) $\forall_{Q} x \forall_{Q} y\left(\operatorname{Fin}(x) \wedge x \subset y \rightarrow q c(x)<_{E} q c(y)\right)$

(Q24) $\forall_{Q} x \forall_{Q} y\left(\forall w(w \notin x \vee w \notin y) \rightarrow q c(x \cup y)={ }_{E} q c(x)+q c(y)\right)$

In the next axiom, $2^{q c(x)}$ denotes (intuitively) the quantity of subquasi-sets of $x$. Then,

(Q25) $\forall_{Q} x\left(q c(\mathcal{P}(x))={ }_{E} 2^{q c(x)}\right)$

If the concept of identity has no meaning for $m$-atoms, how can we ensure that a qset $x$ such that $q c(x)={ }_{E} \alpha$ has precisely $2^{\alpha}$ subqsets? In standard set theories (as in the 'classical part' of $\mathfrak{Q}$, that is, in considering those qsets which fit the sets of ZFU), as is well known, if $\operatorname{card}(x)$ denotes the cardinal of $x$, then by the definition of exponentiation of cardinals, $2^{\operatorname{card}(x)}$ is defined to be the cardinal of the set ${ }^{x} 2$, which is the set of all functions from $x$ to the Boolean algebra $2=\{0,1\}{ }^{33}$ In $\mathfrak{Q}$ this definition doesn't work. Let us explain why.

Suppose that $\alpha$ is the quasi-cardinal of $x$, which is a cardinal by Q19. This axiom says that every qset has an unique quasi-cardinal which is a cardinal (defined in the 'classical part' of the theory), and if the qset is in particular a set (in $\mathfrak{Q}$ ), then this quasi-cardinal is its cardinal stricto sensu. So, every quasicardinal is a cardinal and the above expression 'there is a unique' makes sense.

\footnotetext{
${ }^{31}$ Enderton 1977, p. 222.

${ }^{32}$ See French and Krause 1999.

${ }^{33}$ Enderton 1977, p. 141.
} 
Furthermore, from the fact that $\emptyset$ is a set, it follows that its quasi-cardinal is 0 . Then we may define

$$
2^{q c(x)}={ }_{\mathrm{df}} q c\left({ }^{\alpha} 2\right)
$$

and then, since $\alpha$ is a cardinal and both $\alpha$ and 2 are sets in $\mathfrak{Q}$ (that is, they are copies of ZFU-sets), we have

$$
2^{q c(x)}={ }_{E} \operatorname{card}\left({ }^{\alpha} 2\right) .
$$

So, we may take the cardinal of the qset ${ }^{\alpha} 2$ in its usual sense to mean $2^{q c(x)}$. Then, this last equality gives meaning to the axiom Q25, since it explains what $2^{q c(x)}$ means: it is the cardinal of the set of all the applications from $\alpha$ (the quasi-cardinal of $x$ ) in 2. By considering this, the axiom may be written as follows, where $x$ is a qset and $\alpha$ is its quasi-cardinal:

Axiom Q25 (Alternative Form) $\forall_{Q} x\left(q c(\mathcal{P}(x))={ }_{E} \operatorname{card}\left({ }^{\alpha} 2\right)\right.$.

We remark that the second member of this equality has a precise meaning in $\mathfrak{Q}$, since both $\alpha$ and 2 act as in classical set theories, as remarked above since they are (copies of) sets. This characterization allows us to avoid another problem, which could be thought to be derived in quasi-set theory. To explain it, we recall that in standard set theories we can prove that $\mathcal{P}(x)$ is equinumerous with ${ }^{x} 2$ by defining a one-one function $f: \mathcal{P}(x) \rightarrow 2$ as follows: for every $y \subseteq x$, let $f(y)$ be the characteristic function of $y$, namely, the function $\chi_{y}: x \rightarrow 2$ defined by

$$
\chi_{y}(t)=_{\mathrm{df}} \begin{cases}1 & \text { if } t \in y \\ 0 & \text { if } t \in x-y\end{cases}
$$

Then any function $h \in{ }^{x} 2$ belongs to the range of $f$ since

$$
h=f(\{t \in x: h(t)=1\}) .{ }^{34}
$$

Suppose now that $x$ is a qset such that $q c(x)$ is the natural number $n$ and that all elements of $x$ are indistinguishable from each other (the natural numbers are defined in $\mathfrak{Q}$ in the usual way, just as in the model of ZFU we have defined in $\mathfrak{Q}){ }^{35}$ In this case, we cannot define the characteristic quasi-function $\chi_{y}^{q}$ for $y \subseteq x$, since, for instance, if $\chi_{y}^{q}(t)={ }_{E} 1$ for $t \in y$, then $\chi_{y}^{q}(w)={ }_{E} 1$ as well for every $w \in x$, independently if either $w$ belongs to $y$ or not. This is due to the definition of the quasi-functions given above, since for every quasi-function $f$,

$$
\langle a, b\rangle \in f \wedge\langle c, d\rangle \in f \wedge a \equiv c \rightarrow b \equiv d .
$$

In other words, if the image of a certain $t$ by the quasi-function $f$ is 1 , then the image of every element indistinguishable from $t$ will be 1 as well. So, $\mathfrak{Q}$

\footnotetext{
${ }^{34}$ Enderton op. cit., p. 131.

${ }^{35}$ For all we need, it is sufficient to consider finite qsets (this definition is also standard; roughly speaking, their quasi-cardinals are natural numbers).
} 
distinguishes only between two quasi-functions from $x$ to 2, namely, that one which associates 1 to all elements of $x$ and that one which associates 0 to all of them. This is the motive why we have used $q c\left({ }^{\alpha} 2\right)$ to mean $2^{q c(x)}$, since both $\alpha$ and 2 may be viewed as sets (in the standard sense). If we had used ${ }^{x} 2$ instead, we would be unable to distinguish among certain quasi-functions, so complicating the meaning of Q25, since we could have no way of counting the number of subquasi-sets of a qset. But, by using ${ }^{\alpha} 2$, since both $\alpha$ and 2 behave 'classically', we keep Q25 with its usual meaning.

From these considerations, we may conclude that when $x$ is a qset whose elements are indistinguishable $m$-atoms, we cannot prove within $\mathfrak{Q}$ that if $q c(x)={ }_{E}$ $n$, so we cannot count $2^{n}$ subquasi-sets in $x$. Since this is precisely what Q25 intuitively says, we may affirm that this axiom cannot be proven from the remaining axioms of $\mathfrak{Q}$. But, since it holds for particular qsets, namely, those which are $\mathfrak{Q}$-copies of sets, it cannot be disproved either. In order to show that Q25 cannot be disproved, consider the sets in $\mathfrak{Q}$; since they behave as classical sets, we can prove that what Q25 asserts is true. Now it suffices to take a qset whose elements are indistinguishable $m$-atoms and such that $q c(x)={ }_{E} \alpha$.

Axiom Q25 has another important implication for $\mathfrak{Q}$. In standard set theories, if $\operatorname{card}(x)$ is (say) the natural number $n$, then there are exactly $n$ subsets of $x$ which are singletons. Can this result be proved also in $\mathfrak{Q}$ ? If not, how can we make sense of the idea that if $q c(x)={ }_{E} n$, then $x$ has $n$ elements? We recall once more that the main motivation for $\mathfrak{Q}$ is the way quantum mechanics deals with elementary particles and then, although there is a sense in saying that, say, there are $k$ electrons in a certain level of a certain atom, there is no way of counting them or of distinguishing among them, as we have already remarked (see also below). These considerations motivate the definitions and axioms of the next section. ${ }^{36}$

\subsection{The 'Weak' Extensionality}

If $x$ is a qset whose elements are indistinguishable from one another as above (let us suppose again that $q c(x)={ }_{E} n$, which suffices for our purposes), then the singletons $y \subseteq x$ are indistinguishable from each other, as follows from the Weak Extensionality axiom Q26 below. So, all the singletons (in the intuitive sense) seem to fall into just one qset. But it should be recalled that these 'singletons' (subqsets whose quasi-cardinality is 1) are not identical (that is, they cannot be proven to be the same object in the theory), but they are indistinguishable in a precise sense (given by Q26 below). In other words, although the theory cannot distinguish between them, we cannot affirm either that they are the same qsets or that their elements are identical. So, it is consistent with $\mathfrak{Q}$ to suppose that if $q c(x)={ }_{E} \alpha$, then $x$ has precisely $\alpha$ 'singletons' (which are of course not of the form $[y]$ given above). So, due to Q25, the theory does not forbid the existence of such singletons, despite the fact that in $\mathfrak{Q}$ we cannot prove that they exist as

\footnotetext{
${ }^{36}$ Sant'Anna and Santos 2000 have proposed a modified version of axiom Q25 to cope with other physical insights.
} 
'distinct' entities, and hence we may reason in $\mathfrak{Q}$ as physicists do when dealing with a certain number of indistinguishable quanta or with collections of them.

The absence of a theory of identity for the $m$-atoms, due to the lack of meaning of speaking about either the identity or the difference of $m$-atoms, causes the necessity of a modification in the usual Axiom of Extensionality of standard set theories, ${ }^{37}$ which here does not hold as usual. In order to do so, let us introduce the following definition:

Definition 12 For all non empty quasi-sets $x$ and $y$,

(i) $\operatorname{Sim}(x, y)=_{\mathrm{df}} \forall z \forall t(z \in x \wedge t \in y \rightarrow z \equiv t)$. In this case we say that $x$ and $y$ are similar.

(ii) $Q \operatorname{Sim}(x, y)=_{\mathrm{df}} \operatorname{Sim}(x, y) \wedge q c(x)={ }_{E} q c(y)$. That is, $x$ and $y$ are $Q$-similar iff they are similar and have the same quasi-cardinality.

In the axiom below, $x / \equiv$ stands for the quotient qset of some qset $x$ by the equivalence relation $\equiv$.

(Q26) [Weak Extensionality] Qsets which have the same quantity of elements of the same sort are indistinguishable. In symbols,

$$
\begin{aligned}
& \forall_{Q} x \forall_{Q} y((\forall z(z \in x / \equiv \rightarrow \exists t(t \in y / \equiv \wedge \wedge Q \operatorname{Sim}(z, t)))) \\
& \wedge \forall t(t \in y / \equiv \rightarrow \exists z(z \in x / \equiv \wedge \wedge Q \operatorname{Sim}(t, z))) \rightarrow x \equiv y)
\end{aligned}
$$

It is easy to note that if there are no $m$-atoms involved, once in this case $\equiv$ becomes usual identity, then the axiom coincides with the standard axiom of extensionality used in ZFC.

As a consequence, it is easy to prove the following theorem:

\section{Theorem 13}

(i) $x={ }_{E} \emptyset \wedge y={ }_{E} \emptyset \rightarrow x \equiv y$

(ii) $\forall_{Q} x \forall_{Q} y\left(\operatorname{Sim}(x, y) \wedge q c(x)={ }_{E} q c(y) \rightarrow x \equiv y\right)$

(iii) $\forall_{Q} x \forall_{Q} y(\forall z(z \in x \leftrightarrow z \in y) \rightarrow x \equiv y)$

(iv) $x \equiv y \wedge q c([x])={ }_{E} q c([y]) \leftrightarrow[x] \equiv[y]$

One of the main applications of the Weak Extensionality axiom is the theorem of the Unobservability of Permutations to be presented below, which provides a way of expressing in quasi-set theory the idea that if a certain object is 'permuted' by an indistinguishable one, then 'nothing changes at all'! Of course this has no meaning in standard mathematics, first due to the lack of sense in

\footnotetext{
${ }^{37} \mathrm{As}$ it is well known, this axiom says that those sets which have the same elements are identical.
} 
speaking on indistinguishable but not 'identical' objects, as we have seen elsewhere. Furthermore, the standard axiom of extensionality should act, and hence whatever permutation of non-identical object would give us a different set. In what follows we shall show other applications of this axiom, but before that let us comment on the replacement axioms of $\mathfrak{Q}$.

\subsection{Replacement axioms}

We may add to the quasi set theory replacement axioms as follows. If $A(x, y)$ is a formula in which $x$ and $y$ are free variables, we say that $A(x, y)$ defines a $y$-(quasifunctional) condition on the quasi-set $t$ if $\forall w\left(w \in t \rightarrow \exists s A(w, s) \wedge \forall w \forall w^{\prime}(w \in\right.$ $t \wedge w^{\prime} \in t \rightarrow \forall s \forall s^{\prime}\left(A(w, s) \wedge A\left(w^{\prime}, s^{\prime}\right) \wedge w \equiv w^{\prime} \rightarrow s \equiv s^{\prime}\right)$ ) (this is abbreviated by $\forall x \exists$ ! $y A(x, y))$. Then, we have:

(Q27) [Replacement Axioms]

$$
\forall x \exists ! y A(x, y) \rightarrow \forall_{Q} u \exists_{Q} v(\forall z(z \in v \rightarrow \exists w(w \in u \wedge A(w, z))) .
$$

Intuitively speaking, this axiom says that the images of qsets by quasifunctions are also qsets. It is easy to see that when there are no $m$-atoms involved, that is, when $\mathfrak{Q}$ becomes essentially ZFU, then this schema coincides with standard replacement axioms. The difference here is the way of stating the schema, for we must obey the restriction imposed above to the concept of quasi-function.

\subsection{The strong singleton}

An important concept in quasi-set theory is that of the strong singleton of an element $x$ (either a qset or an atom). This is a qset with quasi-cardinality 1 whose 'only element' is indistinguishable from $x$. It is interesting that, contrary to what would be expected, we cannot prove that this element is $x$. So, we will arrive at a situation according to which we will be able to say that we have just one element of a certain kind but without theoretical means of identifying it, even in principle. Let us look at the details.

Definition $14 A$ strong singleton of $x$ is a quasi-set $x^{\prime}$ which satisfies the following property:

$$
x^{\prime} \subseteq[x] \wedge q c\left(x^{\prime}\right)={ }_{E} 1 .
$$

In words, a strong singleton of $x$, as remarked above, is a qset $x^{\prime}$ whose only element is indistinguishable from $x$. In standard set theories, this qset is of course the singleton stricto sensu whose only element is $x$ itself, but here $x$ may be an $m$-atom, and in this case there is no way of speaking of something being identical to $x$. Even so, we can prove that such a qset exists:

Theorem 15 For all $x$, there exists a strong singleton of $x$. 
Proof: The qset $[x]$ exists by the weak pair axiom. Since $x \in[x]$ (since $\equiv$ is reflexive), we have that $q c([x]) \geq_{E} 1$ by Q20. But, by Q21, there exists a subqset of $[x]$ which has quasi-cardinal 1 . Take this qset to be $x^{\prime}$. I

Theorem 16 All the strong singletons of $x$ are indistinguishable.

Proof: Immediate consequence of Q26, since all of them have the same quasicardinality 1 and their elements are indistinguishable by definition.

The important remark is that, as we shall see, we cannot prove that the strong singletons of $x$ are extensionally identical. With regard indistinguishable $m$-atoms, we cannot give ostensive definitions, say, by putting our finger over an $m$-atom and saying 'This is Peter'. Even so, as in quantum physics, we may reason as if a certain element does or does not belong to the qset; the law of the Excluded Middle $x \in y \vee x \notin y$ remains valid, even if we cannot verify which case holds. ${ }^{38}$ This idea fits what happens with the electrons in an atom, as we have already explained in other parts of this book; in general we know how many electrons there are, and we can say that some of them are in that atom, but we cannot tell which particular electrons are in the atom: this question simply loses its usual meaning.

Theorem 17 For all qsets $x$ and $y$, if $y \subseteq x$ and $x$ is finite, then $q c(x-y)={ }_{E}$ $q c(x)-q c(y)$.

Proof: By definition, $t \in x-y$ iff $t \in x \wedge t \notin y$. Then $(x-y) \cap y={ }_{E} \emptyset$. Hence, by Q25, $q c((x-y) \cup y)={ }_{E} q c(x-y)+q c(y)$ (let us call this expression (i)). But, since $y \subseteq x,(x-y) \cup y={ }_{E} x$ and so, in order for (i) to be true, $q c(x-y)={ }_{E} q c(x)-q c(y)$.

The next result may be regarded as a quasi-set version of the Indistinguishability Postulate, which, as we have noted elsewhere, says that permutations of indistinguishable quanta are not observable. In order to state and prove this result, we introduce a definition. ${ }^{39}$

\section{Definition 18}

(i) Let $x$ be a qset such that $E(x)$, that is (according to Definition 1), its elements are also qsets. Then,

$$
\bigcap x=\mathrm{df}[z \in \bigcup x: \forall s(s \in x \rightarrow z \in s)]
$$

(ii) If $m(u),{ }^{40}$ then $S_{u}=_{\mathrm{df}}[s \in \mathcal{P}([u]): u \in s]$

\footnotetext{
${ }^{38}$ These facts cause interesting consequences in the underlying logic. For instance, the 'choice rule' (Mendelson 1997, p. 81) $\exists x \alpha(x) \rightarrow \alpha(t)$ cannot hold for $t$ being a descriptivist term, like an individual constant, which names an individual; the rule must be written $\exists x \alpha(x) \rightarrow \alpha(y)$ instead, where $y$ is a variable.

${ }^{39}$ The Lemmas that follow were originally formulated and proven by A. Sartorelli.

${ }^{40}$ The case where $u$ is an $m$-atom is of course the most interesting one. The generalization of this definition to $M$-atoms and sets is immediate, but in this case the result coincides with the analogous (and sometimes trivial) situation in standard set theories. The case of $m$-atoms is really the one which makes the difference.
} 
(iii) $u^{*}=_{\mathrm{df}} \bigcap_{t \in S_{u}} t$

Lemma 19 If $m(u)$, then:

(i) $u \in \bigcup_{t \in S_{u}} t$

(ii) $\forall s\left(s \in S_{u} \rightarrow u \in s\right)$

(iii) $z \in u^{*}$ iff $z \in \bigcup_{t \in S_{u}} t \wedge \forall s\left(s \in S_{u} \rightarrow z \in s\right)$

(iv) $u \in u^{*}$

(v) $u^{*} \subseteq[u]$

(vi) If $s \in S_{u}$, then $u^{*} \subseteq s$

Proof: (i) $z \in \bigcup_{t \in S_{u}} t$ iff $\exists t\left(t \in S_{u} \wedge z \in t\right)$. Therefore, by the above definition, $z \in \bigcup_{t \in S_{u}} t$ iff $\exists t(t \in \mathcal{P}([u]) \wedge u \in t \wedge z \in t)$. But since $[u] \in \mathcal{P}([u])$ and $u \in[u]$, it follows that $u \in \bigcup_{t \in S_{u}} t$. (ii) $\forall s\left(s \in S_{u} \leftrightarrow s \in \mathcal{P}([u]) \wedge u \in s\right.$ ). Therefore, $\forall s\left(s \in S_{u} \rightarrow u \in s\right)$. (iii) Immediate consequence of the above definition. (iv) is an immediate consequence of (i)-(iii). (v) Suppose that $z \in u^{*}$. By (iii), we have $\forall s\left(s \in S_{u} \rightarrow z \in s\right)$. But since $[u] \in S_{u}$, it follows that $z \in[u]$. (vi) If $z \in u^{*}$, then, as before, $\forall s\left(s \in S_{u} \rightarrow z \in s\right)$. But, by hypothesis, $s \in S_{u}$; so, $z \in s$.

Lemma 20 If $u$ is an m-atom and $z$ is a qset, then if $z \subseteq u^{*}$ and $q c(z)={ }_{E} 1$, it follows that $u \in u^{*}-z$ or $q c\left(u^{*}\right)={ }_{E} 1$.

Proof: Suppose that $u \notin u^{*}-z$. Since $u \in u^{*}$, it follows that $y \in z$. But $z \subseteq u^{*} \subseteq[u]$, therefore $z \in S_{u}$. But, by the above Lemma (6), $u^{*} \subseteq z$. By hypothesis, $z \subseteq u^{*}$, hence $u^{*}={ }_{E} z$, and so $q c\left(u^{*}\right)={ }_{E} q c(z)={ }_{E} 1$. I

Theorem 21 For every $u, q c\left(u^{*}\right)={ }_{E} 1$.

Proof: By (iv) of Lemma (19), $u^{*} \neq_{E} 0$. So, by Q20, $q c\left(u^{*}\right) \neq_{E} 0$, hence $q c\left(u^{*}\right) \geq_{E} 1$. We shall show that the equality holds. Suppose that $q c\left(u^{*}\right)>_{E} 1$. Then, by Q21, there exists a qset $w \subseteq u^{*}$ such that $q c(w)={ }_{E} 1$. So, by Lemma (20), $u \in u^{*}-w$. But $u^{*}-w \subseteq[u]$, since $u^{*} \subseteq[u]$, therefore, by (v) of Lemma (19), $u^{*} \subseteq u^{*}-w$. But since $u^{*}-w \subseteq u^{*}$, it follows that $u^{*}={ }_{E} u^{*}-w$. Again by Q20, $w \neq{ }_{E} \emptyset$ since $q c(w)={ }_{E} 1$. Then let be $t \in w$. So, $t \in u^{*}$ since $w \subseteq u^{*}$, hence $t \in u^{*}-w$ (once $\left.u^{*}={ }_{E} u^{*}-w\right)$. Then $t \notin w$, a contradiction.

Lemma 22 For all $m$-atoms $u$ and $v$, if $u \equiv v$, then $u^{*} \equiv v^{*}$. Furthermore, if $u \in w$, then $u^{*} \subseteq w$ for any qset $w$.

Proof: If $u \equiv v$, then $u^{*} \subseteq[u]$ and $v^{*} \subseteq[v]$, so $\operatorname{Sim}\left(u^{*}, v^{*}\right)$ (see Definition (12)). But, by Theorem (21), $q c\left(u^{*}\right)={ }_{E} 1$ and $q c\left(v^{*}\right)={ }_{E} 1$ and then, by Lemma (20), $u^{*} \equiv v^{*}$. The last part can be proven by noting that if $u \in w$, then $u \in w \cap[u]$, so as $w \cap[u] \subseteq[u]$, therefore $w \cap[u] \subseteq S_{u}$. Then, by (v) of Lemma (19), $u^{*} \subseteq w \cap[u]$ and so $u^{*} \subseteq w$. I 
These last results show that $u^{*}$ is, as expected, a strong singleton of $u$. The remarkable fact is that we cannot prove that $u^{*} \equiv v^{*}$ entails $u^{*}={ }_{E} v^{*}$. This is due to the fact that nothing in the theory can assure that that $m$-atom that belongs to $u^{*}$ is the same $m$-atom that belongs to $v^{*}$, since neither the expression $u=v$ nor $u={ }_{E} v$ are well formed in this case. Furthermore, it is worth recalling that the usual Extensionality Axiom, which could be used for expressing this fact, is not an axiom of our theory but, instead, we have the 'weak' axiom Q26 which talks about indistinguishability only, but not about identity. The impossibility of proving the above result should not be regarded as a deficiency of the theory, but rather as expressing the fact that it is closer to what happens in quantum physics than usual set theories. The next theorem reinforces this point.

\subsection{Permutations are not observable}

If we think of material bodies as collections of quanta of some sort, as intuitively we may think of that wall in front of us as 'composed' of atoms, protons, electrons and the like, the first attempt for approaching its mathematical characterization is to regard such a collection as a set endowed with some kind of structure. For instance, Walter Noll's definition of a continuum body says that a body is a triple $\langle B, \Phi, m\rangle$ where $B$ is an arbitrary set, $\Phi$ is a set of mappings (hence, also sets) from $B$ into $E$, the three-dimensional Euclidean point space, and $m$ is a function defined on the subsets of $B$ into the set $\Re$ of real numbers; the set $B$ is the set of particles of the continuum body. There follows some axioms which provide the desired 'structure', but which do not interest us here. $^{41}$

Of course we know that a material body is not just a collection of particles, as our body is not a collection (set) of cells. As recalled by Toraldo di Francia, in discussing this point, a physical body is richer than a simple set of objects. As he says, "put together a million billiard balls and try to see if we can observe something interesting". ${ }^{42}$ But even so we could try to approach 'quantum bodies' in Noll's sense. Granted that the analogous definition in quantum theory would be more complicated, a 'quantum body' should still be regarded as a collection of some sort, plus something which expresses the 'structural' characteristics. But to express this structural aspect is far from be a simple task. Of course, it is well known from our knowledge of isomers that collections of 'identical' atoms may yield completely different substances, as for instance $\mathrm{C}_{2} \mathrm{H}_{6} \mathrm{O}$ may stand for both $\mathrm{CH}_{3}-\mathrm{CH}_{2}-\mathrm{OH}$, the ethylic alcohol and $\mathrm{H}_{3} \mathrm{C}-\mathrm{O}-\mathrm{CH}_{3}$, the methylic ether. But, to consider collections is a first step in this direction. Then, setting aside all the details and paying attention just to these collections, even so we find them quite distinct from standard sets. One of the most basic characteristics of such collections (which would stand for the $B$ in Noll's definition) is that certain elements can be exchanged without altering the collection.

\footnotetext{
${ }^{41}$ See Ignatieff 1996, Chap. 9.

${ }^{42}$ Toraldo di Francia 1990, p. 17.
} 
In other words, the Indistinguishability Postulate (IP) should hold and hence such collections should satisfy the basic quantum situation, nicely expressed by Roger Penrose that

"according to the modern theory [QM], if a particle of a person's body were exchanged with a similar particle in one of the bricks of this house then nothing would have happened at all". ${ }^{43}$

The next theorem is the quasi set version of this principle and constitute an important step to see quantum bodies as entities like Noll's bodies.

Theorem 23 [Unobservability of Permutations] Let $x$ be a finite qset such that $x \neq_{E}[z]$ and $z$ an $m$-atom such that $z \in x$. If $w \equiv z$ and $w \notin x$, then there exists $w^{\prime}$ such that

$$
\left(x-z^{\prime}\right) \cup w^{\prime} \equiv x
$$

Proof: Case 1: $t \in z^{\prime}$ does not belong to $x$. In this case, $x-z^{\prime}={ }_{E} x$ and so we may admit the existence of $w^{\prime}$ such that its unique element $s$ does belong to $x$ (for instance, $s$ may be $z$ itself); then $\left(x-z^{\prime}\right) \cup w^{\prime}=_{E} x$. Case 2: $t \in z^{\prime}$ does belong to $x$. Then $q c\left(x-z^{\prime}\right)=_{E} q c(x)-1$ by the above Theorem (17). Then we take $w^{\prime}$ such that its element is $w$ itself, and so it results that $\left(x-z^{\prime}\right) \cap w^{\prime}={ }_{E} \emptyset$. Hence, by Q25, $q c\left(\left(x-z^{\prime}\right) \cup w^{\prime}\right)={ }_{E} q c(x)$. This intuitively says that both $\left(x-z^{\prime}\right) \cup w^{\prime}$ and $x$ have the same quantity of indistinguishable elements. So, by applying Q27 (see above), we obtain the result.

Supposing that $x$ has $n$ elements, then if we 'exchange' their elements $z$ by correspondent indistinguishable elements $w$ (set theoretically, this means performing the operation $x-z^{\prime} \cup w^{\prime}$ ), then the resulting qset remains indistinguishable from the original one. In a certain sense, it is not important, from a pragmatic point of view, if it is either $x$ or $x-z^{\prime} \cup w^{\prime}$ that we are dealing with. If this result can help in some discussion on reidentificability or is a kind of discussion we leave to the reader.

\subsection{The Axiom of Choice}

Finally, the theory $\mathfrak{Q}$ can be supplied with a version of the axiom of choice:

(Q28) [The Axiom of Choice]

$$
\begin{array}{r}
\forall_{Q} x\left(E(x) \wedge \forall y \forall z\left(y \in x \wedge z \in x \rightarrow y \cap z=_{E} \emptyset \wedge y \neq_{E} \emptyset\right) \rightarrow\right. \\
\left.\exists_{Q} u \forall y \forall v\left(y \in x \wedge v \in y \rightarrow \exists_{Q} w\left(w \subseteq[v] \wedge q c(w)=_{E} 1 \wedge w \cap y \equiv w \cap u\right)\right)\right)
\end{array}
$$

Intuitively speaking, if $x$ is a qset whose elements are disjointed non empty qsets, then there exists a qset $u$ such that for every $y \in x$ and $v \in y, u$ has as element an indististinguishable from $v$ (which is expressed by the last part of

\footnotetext{
${ }^{43}$ Penrose 1989, p. 360.
} 
the axiom). In other words, we may form a qset which has elements indistinguishable from the members of the elements of $x$. Using Russell's well known example of the pairs of socks, we are not collecting in the 'choice qset' $u$ one element of each pair of socks, but $a$ sock indistinguishable from one of the elements of each pair of socks (which of course may be one of them, but we can never prove that). This is closer to what happens with bodies in general (and with persons in particular) if we consider what has been said about personal identity; we cannot strictly 'prove' that a certain person we are seeing now is the same one we saw yesterday; as Hume has already shown us, the only we can say us that we have a propensity (or a custom) to call that person we are seeing now with the same name we have used before (Hume 1985). Standard mathematics masks this discussion due to extensionality, but maybe quasi set theory can provide the tools for a new discussion.

Of course the discussion could be further explored in several points. For instance, since the idea of a strong singleton of $x$ gives a qset with just one indistinguishable from $x$, then apparently we could think of using such a qset in whatever sentence involving one $x$. In other words, the existence of such strong singletons could act in the theory as Hilbert's $\epsilon$-symbol, which (as it is well known) entails that the sentence which expresses the axiom of choice is a theorem of standard set theories. So, perhaps some form of the axiom of choice would be a necessary resulting fact of whatever theory involving indistinguishability. But this is a matter for future analysis. Anyway, such responses would require that collections of $m$-atoms could be constructed in the theory, and the above axioms do not postulate the existence of Urelemente. Let us comment on this point a little bit next. Finally, let us remark that we can't agree that the Urelemente can fit adequately quantum objects. Although invariant by the action of automorphisms, Urelemente can be seen as sets of the same rank, and are consequently distinguishable from the 'outside', that is, in a model of 'pure' set theory where ZFU is interpreted (which is possible due to their equiconsistency).

\subsection{Remark on the existence of atoms: the theory $\mathfrak{Q}^{m}$}

The reader for sure has noted that in $\mathfrak{Q}$ we have not postulated the existence of atoms. This is in accordance with the most theories involving Urelemente. But for the purposes of physics it seems that the existence of such entities (so as of collections of them) would be interesting and even necessary. If necessary for particular applications, we can add to our theory an axiom saying explicitly that (say) qsets of $m$-atoms exist; let us call $\mathfrak{Q}^{m}$ such a variant of the above theory. So, its specific axiom would be

[Axiom of $\mathfrak{Q}^{m}$ ] $\exists_{Q} x(x \neq \emptyset \wedge \forall y(y \in x \rightarrow m(y)))$

Of course the existence of such collections should be explored, but we shall not develop the theory $\mathfrak{Q}^{m}$ here. Anyhow, it is patent that specific qsets of $m$-atoms can be obtained if necessary, although we guess that in this case (that is, by considering the theory $\mathfrak{Q}^{m}$ ), the relative consistency to Zermelo-Fraenkel (as described below for $\mathfrak{Q}$ ) cannot be obtained. 


\section{Relative Consistency}

Let us come back to the theory $\mathfrak{Q}$. In the preceding sections we have seen that there is a 'copy' of ZFU in $\mathfrak{Q}$. This proves that if $\mathfrak{Q}$ is consistent, so is ZFU (and, hence, so is ZF) (see Figure 1); now let us see what happens in considering the other way around. We shall see that there is a sense in saying that we can 'mimic' the behaviour of quasi sets in ZF (hence in ZFU). ${ }^{44}$ This of course does not show that all we can do in $\mathfrak{Q}$ can also be done in ZF (or in ZFU), as we shall see below, for in classical mathematics we can't deal with indistinguishable but not identical objects, except if we relativise the discussion to a certain structure. That is to say, the most we can say is that $a$ and $b$ are indistinguishable relative to a structure $\mathfrak{A}$ (for instance, $a$ and $b$ are indistinguishable in $\mathfrak{A}$ if and only if they are invariant under automorphisms of $\mathfrak{A}) .{ }^{45}$ But even in this case $a$ and $b$ are objects of the universe of ZF, hence they are individuals in the sense of obeying the classical theory of identity, despite their distinction can't be done within the structure. In quasi-set theory, the existence of objects which are indistinguishable from the point of view of the whole theory, that is, from the point of view of the whole model of $\mathfrak{Q}$ is allowed, contrary to classical mathematics.

In the remaining of this section, we shall be working in ZFC. Let $m$ be a non empty set and let $R$ be an equivalence relation on $m$. The equivalence classes of the quotient set $m / R$ are denoted by $C_{1}, C_{2} \ldots$ If $x \in m$, define $\widehat{x}=\left\langle x, C_{x}\right\rangle$, where $C_{x}$ is the equivalence class to which $x$ belongs and call $\widehat{m}$ the set of all $\widehat{x}$ with $x \in m$.

Let $X$ be the set $X=\widehat{m} \cup M$, where $\widehat{m}$ is as above and $M$ is a set such that $\widehat{m} \cap M=\emptyset$ and $\operatorname{rank} \widehat{m}=\operatorname{rank} M$.

Then we define a superstructure $\mathbf{Q}$ over the set $X$, called the $Q$-set universe (see Figure 2). As we will see, $\mathbf{Q}$ acts as a 'model' for the quasi-set theory $\mathfrak{Q}$. The definition is as follows:

$$
\begin{aligned}
& Q_{0}={ }_{\mathrm{df}} X \\
& Q_{1}=\mathrm{df} X \cup \mathcal{P}(X) \\
& \vdots \\
& Q_{\lambda}={ }_{\mathrm{df}} \bigcup_{\beta<\lambda} Q_{\beta} \quad \text { if } \lambda \text { is a limit ordinal } \\
& \mathbf{Q}={ }_{\mathrm{df}} \bigcup_{\alpha \in O n} Q_{\alpha} .
\end{aligned}
$$

\footnotetext{
${ }^{44}$ If is left open whether the same can be said concerning the theory $\mathfrak{Q}^{m}$. We guess that this theory cannot be embedded in ZFC.

${ }^{45}$ See Krause and Coelho, forthcoming. Roughly speaking, inside the structure some elements may be invariant by automorphisms, so 'indistinguishable' from the point of view of the structure according to the standard definitions ibid.. But even so they can be distinguished from the outside since in the Zermelo-Fraenkel universe they are always distinguishable, as we have insisted above; for instance, add to the structure the singletons of these elements, which suffice to make the distinction.
} 


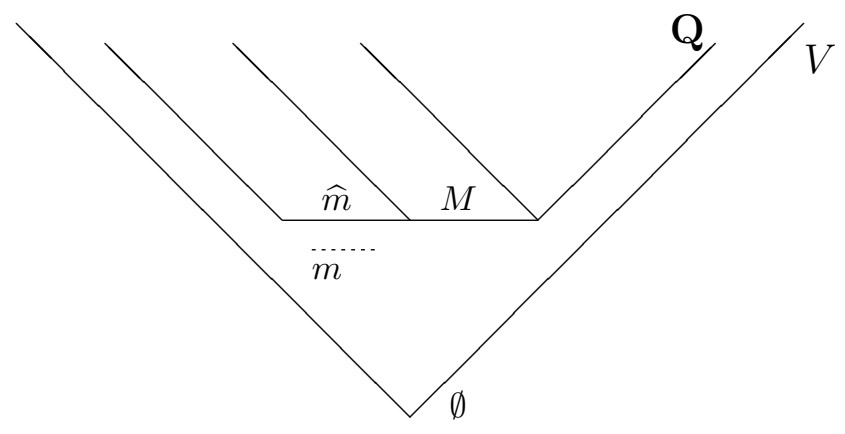

Figure 2: Simulatin qsets in $V$ (the ZFC-universe). The elements of $m$-dashed lines-are outside $\mathbf{Q}$.

In accordance with the terminology of $\mathfrak{Q}$, the elements of $M$ are called $M$ atoms, $M$-elements or $M$-objects, while the elements of $\widehat{m}$ are called $m$-atoms, $m$-elements or $m$-objects. The final goal is to interpret the basic elements of $\mathfrak{Q}$ in the corresponding objects in $\mathbf{Q}$.

For the sake of simplicity, we introduce another superstructure which we will call $\mathbf{Q}^{s}$, constructed in a similar way as $\mathbf{Q}$ above but having only the set $M$ as its 'ground' basis instead of the whole set $X$. The sets of $\mathbf{Q}$ (that is, those $x$ that satisfy the predicate $Z(x)$ ) will be the only elements of $\mathbf{Q}^{s}$.

Now we define a translation from the language of $\mathfrak{Q}$ into the language of ZFC. But firstly let us define on the quotient set $\widehat{m}$ the following relation, which is an equivalence relation, as it is easy to see:

$$
\widehat{x} \sim \widehat{y} \text { iff } C_{x}=C_{y} .
$$

If $\widehat{x} \sim \widehat{y}$, we say that $x$ and $y$ are indistinguishable. We note that this identifys $x$ and $y$ by means of the class (or 'state', or 'sort') they are in, represented by the equivalence classes they belong to, and this is done without direct reference to the objects themselves, which lie outside the structure $\mathbf{Q}{ }^{46}$ This approach is essentially Weyl's idea of 'aggregates' of individuals, a concept he showed to be important in quantum mechanics as well as in chemistry and biology, as we have seen before.

Let us now turn to the translation. Suppose that $A$ is an atomic formula

\footnotetext{
${ }^{46}$ Recall that the rank of the elements of $\widehat{m}$ are lower that the rank of $\widehat{m}$, hence of the elements of $X$. See Figure 2
} 
of the language of $\mathfrak{Q}$; let us call $A^{\prime}$ its translation into the language of ZFC. ${ }^{47}$ We suppose that all those sets (of ZFC) involved in the definition below belong to $\mathbf{Q}$ and that the quantifiers are restricted to this class. Then, the translation says:

(i) If $A$ is $m(x)$, then $A^{\prime}$ is $x \in \widehat{m}$.

(ii) If $A$ is $M(x)$, then $A^{\prime}$ is $x \in M$

(iii) If $A$ is $Z(x)$, then $A^{\prime}$ is $x \in \mathfrak{Q}^{s} \wedge x \notin M$

(iv) The translation of the term $q c(x)$, is $\operatorname{card}(x)$, the cardinal of the set $x$.

(v) If $A$ is $x \equiv y$, then $A^{\prime}$ is $\left.(x \in \widehat{m} \wedge y \in \widehat{m} \wedge x \sim y) \vee x=y\right)$

(vi) If $A$ is $x \in y$, then $A^{\prime}$ is $x \in y$

The other formulas are translated in the usual way. By means of the above procedure, the definitions of $\mathfrak{Q}$ can be translated into ZFC. Let us give some examples:

1. In $\mathfrak{Q}$, a quasi-set is an object which is neither an $m$-atom nor an $M$-atom. The formal definition is $Q(x)={ }_{\mathrm{df}} \neg(m(x) \vee M(x))$, as we have seen. Due to the translation, in ZFC this simply means that $x \in \mathbf{Q}$ but neither $x \in \widehat{m}$ nor $x \in M$. That is, a set, which in ZFC 'represents' a quasi-set, is a set of the class $\mathbf{Q}$ that neither belongs to $M$ nor is an ordered pair of the form $\left\langle x, C_{x}\right\rangle$.

2. In $\mathfrak{Q}$, the 'pure' quasi-sets are those quasi-sets whose elements are only $m$-atoms. In the present case, they are interpreted (in ZFC) as subsets of $\widehat{m}$. Furthermore, in $\mathfrak{Q}$ we define a classical object as an $x$ which obeys the predicate $C$ defined by $C(x)=\mathrm{df}_{\mathrm{d}} M(x) \vee Z(x)$. This simply means that $x$ is either an element of $M$ or of $\mathbf{Q}^{s}$.

3. Quasi-set inclusion is defined as in ZFC; ${ }^{48}$ so, its translation coincides with the standard inclusion of sets in such a theory.

4. Extensional Equality expresses (in ZFC) the usual identity governed by the axiom of extensionality (in the case of sets) or the identity of the elements of the set $M$.

Now we should turn to a detailed translation of the axioms of $\mathfrak{Q}$ so that the translated formulas can be proven to be theorems of ZFC. Those axioms of $\mathfrak{Q}$ which are adaptations of the axioms of ZFU have obvious translations. ${ }^{49}$ We remark that, in $\mathfrak{Q}$, the Axioms of Indistinguishability state that $\equiv$ has the properties of an equivalence relation. If we consider the above translation,

\footnotetext{
${ }^{47}$ We use $x, y, \ldots$ as individual variables in both theories.

${ }^{48}$ That is, $x \subseteq y$ iff $\forall z(z \in x \rightarrow z \in y)$.

${ }^{49}$ In the present case, the elements of $X$ play the rôle of the Urelemente in Q: the elements of $\widehat{m}$ act as the $m$-atoms while those of $M$ act as the atoms in ZFU.
} 
it is easy to see that the images of the pairs $\langle x, y\rangle$ such that $x \equiv y$ define an equivalence relation in ZFC. ${ }^{50}$ Concerning the extensional identity, since it means no more than the usual identity for certain sets of ZFC, it results that the substitutivity law is also valid. So, the translations of the axioms (Q1) throughout (Q4) are true in ZFC.

The other axioms of $\mathfrak{Q}$ and their translations can be dealt with according to the above definitions. Then, by careful checking, we can realize that all these translations are true in the defined model. ${ }^{51}$

What is the meaning of this result? We have constructed in ZFC a kind of internal model for quasi-set theory. Hence, if ZFC is consistent, so is $\mathfrak{Q}$. Does this result entail that quasi-set theory is not necessary and that all can be done within classical mathematics, so apparently contradicting what we have said above? Of course this could be said, but it would be a complete misleading, a wrong interpretation of all we have done. Let us explain a little bit what is going on.

An important distinctive feature of the 'model' $\mathbf{Q}$ is that the rank of the sets $x$ and $y$ are smaller that the rank of the elements of the set $X(X=\widehat{m} \cup M)$ which is the ground set of the structure. ${ }^{52}$ In other words, $x$ and $y$ (being elements of the set $m$ ) are outside the model $\mathbf{Q}$; hence, we cannot talk about neither their identity nor about their diversity within $\mathbf{Q}$, so respecting the basic idea involving quasi-sets. But of course we can do this from the outside, for instance in the the standard model $V=\langle V, \in\rangle$ of ZFC, in the same vein as we can talk about the identity and about the diversity of two electrons in the natural language (say, English), despite this does not make much sense in quantum mechanics, as we have seen. ${ }^{53}$ But when we look inside $\mathbf{Q}$ and ask for its internal logic, then of course it cannot be classical logic. This kind of logic we call non-reflexive, meaning a logic in which the 'traditional' (Leibnizian) theory of identity of classical logic does not hold in full. The meaning of this expression 'internal logic' can be seen exactly in the same sense as when one asks for the internal logic of a topos in category theory. There, as it is well known, it is said that such a logic is intuitionistic logic, since the partially ordered sets of 'sub-objects' of a given 'object' 54 is not a Boolean algebra, but a Heyting algebra. ${ }^{55}$ That what happens with collections of indistinguishable things, like quantum objects, is in a certain sense analogous to what happens with toposes, although in the first case we are dealing with empirical sciences, while in the second just with mathematical objects. But a remark made by Hatcher can be applied here; paraphrasing him, we may say that we can study quasi-sets (as collections of

\footnotetext{
${ }^{50}$ In saying that, of course we intend to say that the properties which define an equivalence relation hold for the defined relation. From now on, we shall not make more qualifications like this one, asking for the reader's patience concerning the language we use.

${ }^{51}$ An example of this work for an early version of the quasi-set theory is given in da Costa and Krause 1999.

${ }^{52}$ Let us recall that the rank of a set $A$ is the least ordinal $\alpha$ such that $A \subseteq V_{\alpha}$.

${ }^{53}$ As Schrödinger says, this talk should be understood as "an abbreviation of speech" Schrödinger 1952, p. 17.

${ }^{54}$ This terminology came from category theory.

${ }^{55}$ See Hatcher 1982, p. 303.
} 
non-individual quanta) from the set-theoretical point of view, as the physicist does when he uses classical mathematics. But this is the external logic, which can be chosen to be, as Hatcher says, "anything we choose it to be"; 56 but the internal logic of quasi-set theory (hence, of the structure $\mathbf{Q}$ ) is a kind of non-reflexive logic, in the sense suggested in this chapter. ${ }^{57}$ Furthermore, if we consider the theory $\mathfrak{Q}^{m}$ introduced above, which postulate the existence of qsets of $m$-atoms, the above translation doesn't work, and we guess that no translation from (the languages of) $\mathfrak{Q}$ to ZFC can be defined at all. So, if this is the case, $\mathfrak{Q}^{m}$ is not equivalent to ZFC. The search of such a translation is still an open problem.

Anyhow, even keeping within $\mathfrak{Q}$, if someone would like to dismiss it by saying that due to the above result it doesn't differ from ZFC, we recall that intuitionistic logic has also an interpretation in modal logic S4, but for sure we will not say that intuitionistic logic has not its intrinsic merits due to this fact. We shall show other kind of advantages of $\mathfrak{Q}$ in the next sections. But before that, let us have a contact with quaset theory, for it may helps in fixing the ideas.

\section{Quaset ideas in Quasi-Set Theory}

As we have mentioned in the Introduction, M. L. Dalla Chiara and G. Toraldo di Francia have proposed a quaset theory with the aim of discussing controversial issues involving semantics for the languages of scientific theories about 'the infinitely large' (astrophysics) and 'the infinitely small' (microphysics). Here, we shall make some few remarks on their ideas regarding microphysics only. ${ }^{58}$

Some of the basic facts of microphysics, as the authors observe, are the following (here mentioned without justification): ${ }^{59}$ in microphysics, there are no proper names, because the objects of this domain are described only nomologically, that is, they are given by physical law, in the sense that they have fixed and prescribed characteristics. Contrary to standard 'usual' objects of our surroundings, to which we attribute properties after analysis, the objects of microphysics, to which we have no 'direct' access, so to say, are born with theory, having their characteristics given by the laws of physics. It is a fundamental principle of quantum mechanics, they say, that two micro-particles of the same kind (e.g., two electrons) are absolutely indistinguishable in their physical properties (which do not include location in space-time). According to them, two such particles cannot have 'names' that act as rigid designators. In this realm, there is no trans-world identity, which means that the very notion of a rigid designator becomes completely useless, or fuzzy at least.

\footnotetext{
${ }^{56}$ Op. cit., p. 303.

${ }^{57}$ The terminology 'non-reflexive' came from the fact that since $x=y$ is in general not a formula, the reflexive law of identity (sometimes called Principle of Identity) $\forall x(x=x)$ does not hold at all.

${ }^{58}$ For a detailed review, see Cocchiarella 1995.

${ }^{59}$ The interested reader should have a look in their papers mentioned in the references. See also French \& Krause's forthcoming book.
} 
The notion of a quaset is introduced to deal with the idea that electrons of a particular atom have a cardinality but, since they are indistinguishable, it is not possible to count (or well-order) them. So, the authors introduce the notion of a quaset, which is a collection of elements that are to be indistinguishable from (but not identical to) one another. A quaset, according to them, sometimes can "mimic some properties of a classical body", namely, when are situated in isolation in space and, in this case, can bear a name, although such a name has nothing to do with substantiality. The basic underlying idea is that entities like electrons, as a natural kind, can be characterized intensionally (through a theoretical description of the properties electrons have essentially, that is, nomologically), but not extensionally, or at least, "not by giving the term 'electron' its extension in an ordinary way", as they say. According to them, what we find in nature are electron quasets and not electrons proper, which means that "the extension of the natural kind 'electron' can be defined as a quaset whose subquasets are all the electron systems that can be detected experimentally". In this regard, "the intension of a particle term determines - at least in principlean extension of the term", and we arrive at a situation that is "usually believed to be impossible in classical semantics: different extensions can correspond to one and the same intension".

Let us have a general look on their quaset theory, here not presented in its original formulation.

\subsection{The quaset theory}

The theory QST to be described below is a slight modification from the original one, already involving some terminology taken from quasi-sets (for instance, we use the term 'quasi-cardinal'); the formulation below is based on Dalla Chiara, Giuntini \& Krause's paper (1998). QST is formalized in classical first order logic with identity (which is dealt with as a logical constant), and its language contains the following primitive non-logical concepts:

(i) One monadic predicate: urobject $(O)$;

(ii) Three binary predicates: the positive membership relation $(\in)$, the negative membership relation $(\notin)$, and the inclusion relation $(\subseteq)$. In the intuitive interpretation , $x \in y(x \notin y)$ is read as ' $x$ certainly belongs to $y$ ' (' $x$ certainly does not belong to $y$ ') (see the explanation below);

(iii) A unary functional symbol: the quasi-cardinal of (qcard);

(iv) A binary functional symbol: the quaset-theoretical intersection ( $\square)$.

Restricted quantifiers are assumed as in the theory $\mathfrak{Q}$ (although with different predicates). Further, the quantifier 'there exists exactly one' ( $\exists$ !) is understood as defined in the usual way.

We will present here only the minimal axiomatic nucleus of the theory. The notion of quaset is defined as follows: 
Definition 24 (Quaset) A quaset is something that is not an urobject. In symbols,

$$
Q(x):=\neg O(x) .
$$

Then, the basic idea of a quaset is that of a collection of objects which has a well-defined cardinal, but in such a way that there is no way to tell (with certainty) which are the elements that belong to the quaset. The primitive predicates $\in$ (the positive membership) and $\notin$ (the negative membership, which is not the negation of the former) help in expressing this. The postulates, to be stated below, imply that $z \in y$ entails $\neg z \notin y$, but not the converse. So, it may be the case that it is false that $z$ certainly does not belong to $y$, but this does not entail that $z$ (certainly) belongs to $y$. The elements $z$ for which it may be said that 'it is false that they certainly do not belong to $y$ ' might act as members in potentia of $y .{ }^{60}$ Since the cardinal of the quaset is well defined, there is a kind of 'epistemic' indeterminacy with respect to the elements of a quaset. ${ }^{61}$ The postulates below provide the grounds for the whole theory:

Ax1 All that has elements is a quaset: $\forall x \forall y(y \in x \rightarrow Q(x))$.

Ax2 The inclusion relation $\subseteq$ between quasets is a partial order (reflexive, symmetric and transitive).

In the intended interpretation, $\subseteq$ has an intensional meaning: $x \subseteq y$ can be read as 'the concept $x$ involves (or implies) the concept $y$ '. ${ }^{62}$

Ax3 Suppose that something certainly does not belong to a given quaset. Then it is not the case that it certainly belongs to our quaset, but generally not the other way around: $\forall x \forall y(x \notin y \rightarrow \neg x \in y)$.

As a consequence, a strong tertium non datur principle $(x \in y \vee x \notin y)$ fails, and this opens the door for indetermined membership relations (as they say, "in accordance with the quantum uncertainty relations (op. cit.)).

Ax4 Intensional inclusion implies extensional inclusion (but not the other way around):

$$
\forall x \forall y(x \subseteq y \rightarrow \forall z(z \in x \rightarrow z \in y) \wedge(z \notin y \rightarrow z \notin x))
$$

Ax5 Any quaset has exactly one quasiextension, where the quasiextension of a quaset $x$ is the unique quaset that certainly contains all the elements of $x$ and certainly does not contain all the other entities:

$$
\forall_{Q} \exists !_{Q} y \forall z((z \in y \leftrightarrow z \in x) \wedge(z \notin y \leftrightarrow \neg z \in x))
$$

Axiom 5 justifies the definition of a unary functional symbol ext (the quasiextension of $x$ ). - Definition 3.2

\footnotetext{
${ }^{60}$ Dalla Chiara and Toraldo do Francia do not use this terminology.

${ }^{61}$ This makes a distinction to quasi-sets, where the indeterminacy is ontological, in the sense that the objects being considered (the $m$-atoms are in some sense veiled; see Krause \& French 1999.

${ }^{62}$ See also Dalla Chiara 1987.
} 


\section{Definition 25 (The quasi-extension of a quaset)}

$$
\forall x \forall y(y=\operatorname{ext}(x) \leftrightarrow \forall z(z \in y \leftrightarrow z \in x) \wedge(z \notin y \leftrightarrow \neg z \in x))
$$

Definition 26 Sets are quasets that are identical with their quasiextension.

One can easily show that sets satisfy the Extensionality Principle. The extension of an empty quaset (which turns out to be trivially a set) is postulated.

Ax6 [The empty quaset] There exists a quaset that necessarily does not contain any element: $\exists_{Q} y \forall x(x \notin y)$.

$Q S T$ contains a copy of ZF (Zermelo-Frankel set theory), obtained by restricting the the universe of quasets to sets only. For any formula $A$ of ZF, let $A^{z}$ be the corresponding formula of $Q S T$ relativized to sets. Then,

Ax7 If $A$ is any instance for an axiom of $Z F$, then $A^{z}$ is an axiom of $Q S T$.

The notion of quasicardinality of a quaset is introduced as follows:

Ax8 Any quaset has a unique quasicardinal, which is a cardinal number:

$$
\forall x \exists ! y(\operatorname{card}(y) \wedge q \operatorname{card}(x)=y)
$$

A similar remark we have made concerning quasi-sets is in order here in regarding quasi-cardinals and ordinals, for (roughly speaking) since the quasicardinal of a quaset, being a 'standard' cardinal, is an ordinal, and hence quasets do have ordinals. Dalla Chiara and Toraldo di Francia do not discuss this question, but we think that the same remarks we have done with respect to quasi-sets apply also here, namely, that the corresponding ordinal is not something which can be described by the axioms of the theory. It might exist only 'outside' quaset theory, but this point of course needs explanation.

Ax9 Quasicardinals and cardinals coincide for sets:

$$
\forall x(Z(x) \rightarrow q \operatorname{card}(x)=\operatorname{card}(x))
$$

Ax10 The quasicardinal of a subquaset is less or equal than the quasicardinal of the whole quaset:

$$
\forall x \forall y(x \subseteq y \rightarrow q \operatorname{card}(x) \leq q \operatorname{card}(y))
$$

Ax11 The quasicardinal of a quaset is greater or equal than the quasicardinal of its quasiextension:

$$
\forall x(q \operatorname{card}(x) \geq q \operatorname{card}(\operatorname{ext}(x)))
$$

Ax12 $\sqcap$ represents a weak conjunction for quasets. This weak conjunction coincides with the usual set-theoretical intersection in the case of sets:

$$
\forall x \forall y((x \sqcap y \subseteq x \wedge x \sqcap y \subseteq y) \wedge(Z(x) \wedge Z(y) \rightarrow x \sqcap y=x \cap y))
$$


As a consequence, a separation procedure may be applied. Notice that our axioms do not require that proper quasets (that are not sets) exist. From an intuitive point of view, the quasiextension of a proper quaset does not represent an adequate semantic counterpart for the usual notion of extension. Think for instance of the fact that the quasiextension a quaset, whose quasicardinal is greater than 0 , might have an empty quasiextension.

It should be of course interesting to follow Dalla Chiara and Toraldo di Francia's use of quasets in their semantic analysis of physics, but this will be not made here. Let us focus just on a minor but interesting point: what can be said about the validity of Leibniz Principle in this theory? Are non-identical objects always distinguished by a property, represented by a quaset? As expected, the answer is in the negative. Namely, the implication

$$
\neg x=y \rightarrow \exists_{Q} z(x \in z \wedge y \notin z)
$$

generally fails, and so the classical set-theoretical argument founded on the theorem of ZF:

$$
\neg x=y \rightarrow x \in\{x\} \wedge y \notin\{x\}
$$

cannot be repeated here, for nothing guarantees that the singleton of $x$ (which should be the characteristic property of $x$ ) exists and that $x$ certainly belongs to it.

\subsection{Quasets within $\mathfrak{Q}$}

As we have seen, in quaset theory there are two basic membership relations; $x \notin y$ is read ' $x$ certainly does not belong to $y$ ' and $x \in y$ is read ' $x$ certainly belongs to $y$ '. The interesting fact, as we have seen, is that $x \notin y$ is not the same as $\neg(x \in y)$. Here, to avoid difficulties with the terminology, we shall introduce the following definitions, given in $\mathfrak{Q}$.

Definition $27 x \varepsilon y={ }_{\mathrm{df}} \exists z(z \in y \wedge z \equiv x)$

So, its negation $x / \varepsilon y$ reads $\forall z(x \in y \rightarrow z \not \equiv x)$. In this case, following the terminology of quaset theory, we say that $x$ certainly does not belong to $y$, and that it certainly belongs to $y$ otherwise. Intuitively, we may say that when $z \varepsilon y$, there are 'traces' of $x$ in $y$, for there are indistinguishable from $x$ in $y$. By using the above concept, we can introduce something like the dual of the quasiextension of a quaset; there, the quasi-extension of a quaset $x$ was defined as the collection of all objects that certainly belong to $x$. Now, we can introduce what we can call the fuzzy complement of $x$ relative to a certain previously given quasi-set $z$ (such that $x \subseteq z$ ) to indicate the collection (quasi-set) of the elements of $z$ for which we cannot affirm that they certainly do not belong to $x$; if we call $C l_{z}(x)$ such a quasi-set (the cloud of $x$ relative to $z$ ), then:

Definition 28 (The Cloud of $x$ relative to $z$ ) $C l_{z}(x)={ }_{\mathrm{df}}[t \in z: t \varepsilon x]$ 
The elements of $z$ for which we can't ensure that they certainly do not belong to $x$ may be said to be 'elements in potentia' of $x$, as in Dalla Chiara and Toraldo di Francia's quaset theory, as we have mentioned earlier. This qset $z$ may also be said to be the environment of $x$, that is, the 'place' from where $x$ can exchange its elements. We remark that since $x$ may be a subqset of distinct $z$ 's, by making he variable $z$ to range on a certain collection of qsets, the quasicardinal of the cloud of $x$ remains undetermined. ${ }^{63}$ Furthermore, we can define a quasi-function $a^{\dagger}: z \mapsto z$ as follows: $a^{\dagger}$ associates to $w$ a qset whose elements are indistinguishable from the elements of $x$ and whose quasi-cardinal is $q c(w)+1$, which can be done for considering $w$ the cloud of some $x$ for suitable $z$ 's. In the same way, we may define a quasi-function $a$ which intuitively decreases the quasi-cardinal of $x$ of one unity, among other possibilities. It is clear that these quasi-functions act as the creation and the annihilation operators in quantum field theory, but this parallel shall be not pursued here.

It is easy to prove for instance the following results:

Theorem 29 If $x \subseteq z$, then:

(a) $\forall_{Q} x \forall_{Q} z\left(C l_{z}(x)={ }_{E} \bigcup_{t \in x}[t] \cap z\right)$

(b) $C l_{z}(\emptyset)={ }_{E} \emptyset$ and $C l_{\emptyset}(x)={ }_{E} \emptyset$

(c) For every $z, Z(x) \rightarrow C l_{z}(x)={ }_{E} x$

The concept of the cloud of a qset suggests the idea that a qset $x$ is something in between its extension $\operatorname{Ext}(x)$, namely, the qset of the objects that certainly belong to $x$ and its cloud $C l_{z}(x)$ for some $z$. Due to the fact that we can't identify the elements of a pure qset, we may say that a qset is not strictly determined by its elements, hence, some degree of intensionality is also present here, as remarked by Dalla Chiara and Toraldo di Francia in regarding their quasets.

The concept of the cloud of $x$ may be useful also for the understanding of some basic facts about quasi-sets, since it gives us an interpretation of what means to say that a certain non-individual belongs to a certain collection of entities. Think for instance of a finite quasi-set $x$ whose elements (by simplicity) are indistinguishable $m$-atoms. Then the axioms of the theory $\mathfrak{Q}$ tell us that there is a quasi-cardinal which stands for the number of elements of $x$. But since these elements don't have individuality, how can we verify if a certain object, say $t$, either belongs to $x$ or not? First of all, we should remark that $t$, as used here, cannot be understood as a proper name of an $m$-atom, for we can't reason in quasi-set theory according to our standard ('classical') ways of reasoning; one thing is to discuss the concepts in the metalanguage, which is 'classical', and where we can talk about 'this' or 'that' $m$-atom. But in order to be precise, we need to look at the right formulas written in the language of $\mathfrak{Q}$. In the present case, to say that (a certain) $t$ does belong to $x$ is to say that there is something

\footnotetext{
${ }^{63}$ This can be useful in discussing quantum field theories, that is, when there are 'virtual' particles involved.
} 
like $t$ in $x$ which behaves as we expect $t$ do behave: in our terminology, we say there is an element indistinguishable from $t$ in $x$, that is, $t \varepsilon x$.

The above theorem about the Unobservability of Permutation also helps in fixing this interpretation. Let us recall that this theorem says intuitively that if we exchange an element of $x$ with an indistinguishable one, then the resulting qset is indistinguishable from the original one. The axioms of $\mathfrak{Q}$ state that the membership relation $\in$ behaves as in standard set theories, contrary to what happens in the theory of quasets, but due to the non-individuality of the $m$-atoms, we cannot have, say, a decision procedure (even for finite sets) for checking either a certain element does belong to a qset or not. The axioms act always in the conditional form: if a certain object belongs to a certain qset, then this or that. The concept of the cloud of a qset expresses the idea of a collection (the Cloud) of those elements of which it is false to say that they certainly do not belong to the considered quaset.

Since the $m$-atoms do not have individuality, then there is still a certain epistemic indeterminacy whether a certain element belongs to a certain qset or not; all we can say is that there may be traces of something which behaves like such an element in the collection, but we never can say that we are talking about that element. We have always elements (this is particularly important for nonindividual entities) which 'could be' in $x$, but we cannot affirm that they really are or are not elements of $x$, since there may be elements indistinguishable from them in $x$ which play the same role. In quasi-set theory, as in quaset theory, the quasi-cardinal of a quasi-set is well determined, but now, since the quasi-set $x$ may be a subset of some $z$, then we can't affirm the same about the quasi-cardinality of the cloud of $x$, except if we fix the qset $z$. But, if in the expression $\mathrm{Cl}_{z}(x)$ the variable $z$ remains free, then the quasi-cardinal of the collection remains undetermined.

Of course, the idea of the cloud of $x$ may suggest several applications, but in what follows we shall explore the idea that the quasi-cardinal of a quasi-set may be not well defined. Perhaps there is a link here with the idea of virtual particles and with quantum field theory, as antecipated by Toraldo di Francia, ${ }^{64}$ although he has spoken in terms of quasets. Really, in quantum field theories even the quantity of elements in a certain state might be undermined due to the creation/annihilation processes, so in certain situations even the cardinal of the collections might be not defined (see below). To give a short account to such a situation, let us modify the theory $\mathfrak{Q}$ by exchanging the axioms for quasi-cardinals in order to give a sense in saying that the quasi-cardinal of a quasi-set may vary in time. We shall to it in the next section.

\section{Changes in Time: the theory $\mathfrak{Q}^{t}$}

The intended interpretation of the $m$-atoms of our theory as elementary particles gives the idea that the qsets would stand for certain 'states' in which these particles might be. Of course this is a problematic question, for we need to

\footnotetext{
${ }^{64}$ Toraldo di Francia 1978.
} 
explain the meaning of the term 'state', and this is not so easy to do, even in quantum theory. But even without a detailed discussion, some aspects of this idea might be captured by our formalism and in describing this point we will have the opportunity of mentioning some of the philosophical and mathematical difficulties one has to describe in 'set-theoretical' terms collections of particles when other assumptions are considered, as for instance, the existence of virtual particles.

Dalla Chiara and Toraldo di Francia have suggested that the cardinality of a quaset may vary in time, although they have not developed such an idea. Of course the same might be said for the quasi-cardinal of a quasi-set. Let us consider this possibility in this section by considering how it is possible to map 'the changes in time' of Weyl's aggregates ${ }^{65}$ into the scope of quasi-set theory.

Hermann Weyl has considered the mathematical treatment of finite collections of objects so that, intuitively speaking, each one of the elements may be in a certain 'state' and that the only information we may have concerns the number of elements of the whole collection (the aggregate) there are in each state, but so that there is no possibility of identifying the elements that belong to each particular state. The fundamental idea concerning Weyl's 'effective aggregates' cannot be accurately described within the scope of classical theory of sets since a set is a collection of distinguishable objects and, so, it should not be possible to maintain the idea that the elements that belong to a certain state should be not (even in principle) distinguished from one another.

In our theory, the effective aggregates in Weyl's sense may be considered as qsets $x / \equiv$ (the quotient of $x$ by the indistinguishability relation), where $x$ is a pure qset, that is, a qset containing only $m$-atoms as elements. That is to say, the states can be viewed as the equivalence classes of elements of $x$ by the indistinguishability relation; since the full concept of identity lacks sense for the $m$-atoms, only their quantity in each 'state' (that is, the quasi-cardinality of each equivalence class) may be known, and this conforms to Weyl's intuitive ideas.

But in doing so we are considering such aggregates only 'statically', that is, without considerations regarding the possibility that the quantity of elements in a state may vary in time. However, Weyl did consider this possibility:

"As long as elements are capable of discrete states only, we are forced to dissolve time into a succession of discrete moments,

$$
t=\ldots,-2,-1,0,1,2, \ldots .
$$

Transition of the system from its state at the time $t$ into its state at time $t+1$ will then be a jump-like mutation. With the $n$ elements individualized by the labels $p$, the changing state of affairs will be described by giving the state $C(x ; t)$ of the element $p$ at the time $t$ as a function of $p$ and $t$. This 'individual' description, by means of the function $C(x ; t)$, is to be supplemented by the principle of relativity

${ }^{65}$ Weyl 1949 , App. B; see also Krause 1991. 
according to which the association between the individuals and their identification marks $p$ is a matter of arbitrary choice; but it is an association for all time, and once established it is not to be tempered with. If, on the other hand, at each moment attention is given to the visible states only, then the numbers $n_{1}(t), \ldots, n_{k}(t)$ in their dependence on $t$ contain the complete picture -however incomplete this information is from the 'individualistic' standpoint. For now we are told only how many elements, namely $n_{i}(t)$, are found in the state $C_{i}$ at any time $t$, but no clues are available whereby to follow up the identity of the $n$ individuals through time; we do not know, nor is it proper to ask, whether an element that is now in the state, say $C_{5}$, was a moment before in the state $C_{2}$ or $C_{6}$. The world is created, as it were, anew at every moment, no bond of identity joins the beings present at this moment with those encountered in the next. This is a philosophical attitude towards the changing world taken by the early Islamic philosophers, the Mutakallimûn. This non-individualizing description is applicable even if the total number $n_{1}(t)+\cdots+n_{k}(t)=n(t)$ of elements does not remain constant in time". ${ }^{66}$

It is possible to map these ideas into the scope of our formalism if we modify conveniently the theory $\mathfrak{Q}$. Let us call $\mathfrak{Q}^{t}$ the theory obtained by modifying the language of $\mathfrak{Q}$ as follows. The new language encompasses the following:

(i) two additional binary predicate symbols: $<$ and $=_{t}$;

(ii) a binary functional symbol: tcard;

(iii) a ternary predicate symbol: $\epsilon$;

(iv) a denumerably infinite collection of variables of 'second species': $t^{\prime}, t^{\prime \prime}, \ldots$. The individual variables of the language of $\mathfrak{Q}$ will be called variables of first species and the functional symbol $q c$ does not appear in this new language.

We use $x, y, \ldots$ and $t, t_{1}, \ldots$ as syntactical variables ranging over the collections of variables of first and of second sort respectively. The variables of second kind are called 'time variables'.

Among the terms, we have now the following additional ones: if $x$ and $t$ are variables of first and of second species respectively, then $\operatorname{tcard}(x, t)$ is also a term.

The set of atomic formulas is increased by the following, where $x$ and $y$ are variables of first species and $t_{1}$ and $t_{2}$ are variables of second species: $\epsilon(x, y, t)$, $t_{1}<t_{2}$ and $t_{1}={ }_{t} t_{2}$.

The formulas in general are defined by the usual procedures, by observing that we now are dealing with a two sorted language. The new terms and atomic formulas may be intuitively thought of in the following sense: $t_{1}<t_{2}$ says that

${ }^{66}$ Weyl op. cit., pp. $242-3$ 
time $t_{1}$ precedes time $t_{2}$ in the order $<; \epsilon(x, y, t)$ will be written $x \in^{t} y$ and means that $x$ is an element of $y$ at the time $t$. Finally, the term $\operatorname{tcard}(x, t)$ stands for the quasi-cardinal of the quasi-set $x$ at the time $t$.

The postulates of $\mathfrak{Q}^{t}$ are essentially those of $\mathfrak{Q}$ (alternatively, we could use $\mathfrak{Q}^{m}$ instead), but instead of the axioms for $q c$, we have the similar axioms for tcard (see below), plus the following ones (we recall that by the definition of atomic formulas, $<$ and $=_{t}$ must be flanked by time variables only):

(T1) $<$ is irreflexive, anti-symmetric and transitive

(T2) $=_{t}$ is reflexive and obeys the substitutivity principle

These axioms impose the usual interpretation on the time relations, and time may be thought of as a succession of discrete moments. (T2) says that $={ }_{t}$ is like classical identity for instants of time. Then, a time variable $t$ may be thought of as ranging over a totally ordered set of instants of time. Obviously, alternative approaches are possible; for instance, instead of a totally ordered set (which may be discrete), we could postulate that the set of instants of time is a complete ordered field, as for instance the set of reals. In this way, we could talk about an 'interval' of time in the usual intuitive sense, as for instance a closed interval of the real number line. For such an alternative, it is sufficient to change the axioms (T1) and (T2) above by the axioms for a complete ordered field, plus an obvious adaptation of the language.

The axioms for the concept of tcard are essentially those we used for $q c$, with adaptations to the case we are considering here, that is, the fact that the primitive concept of cardinal is now a binary functional symbol whose first and second arguments must be occupied respectively by variables of first kind and by time variables. We recall that $\operatorname{card}(x)$ stands for the cardinal of the set $x$, defined in the 'classical part' of the theory, $\exists$ ! means 'there is exactly one', $C d(y)$ stands for ' $y$ is a cardinal' (also defined in the qset copy of ZFU), $[x]$ denotes the qset of the objects which are indistinguishable from $x$ (which in general has more than one element) and $\mathcal{P}(x)$ is the power-qset of $x$. The axioms are the following:

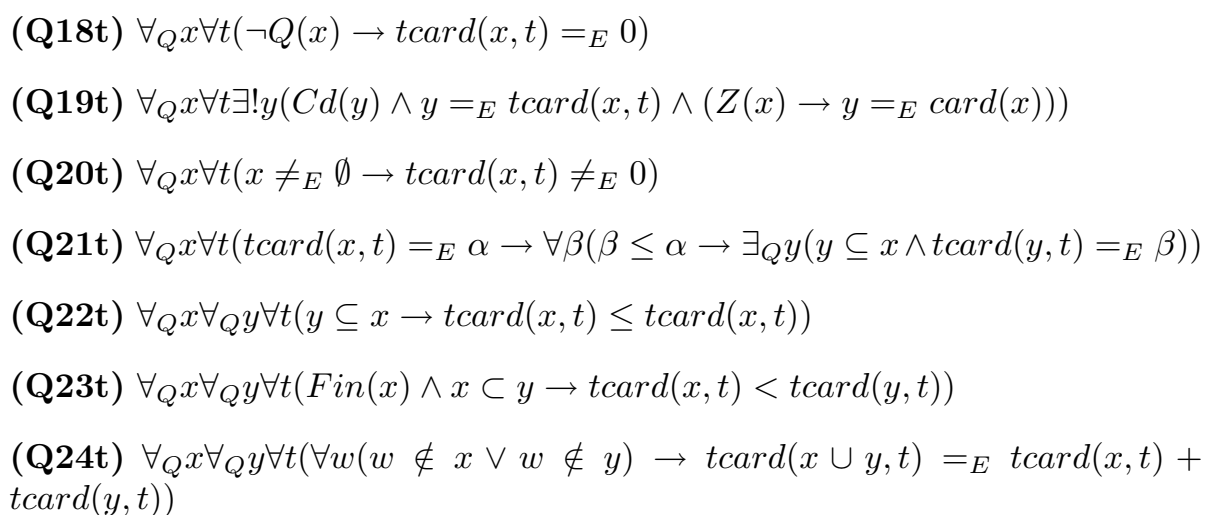




$$
\text { (Q25t) } \forall_{Q} x \forall t\left(\operatorname{tcard}(\mathcal{P}(x), t)={ }_{E} 2^{\operatorname{tcard}(x, t)}\right)
$$

The above axioms, as it is easy to see, preserve all the desired properties of the quasi-cardinal of a qset (which, by force of Q19t, is also a cardinal) in each instant of time. Then, Weyl's aggregates are qsets $x / \equiv$, where $x$ is a pure qset and $\equiv$ our indistinguishability relation, but now the quasi-cardinal of the equivalence classes may vary in time.

A link with our previous considerations can be given as follows. Suppose that the qset $z$ in the definition of the cloud of $x$ relative to $z$ may vary, that is, $z$ range on the sequence of qsets $z^{\prime} \subseteq z^{\prime \prime} \subseteq z^{\prime \prime \prime} \ldots$. We can associate this sequence with a time sequence $t, t^{\prime}, t^{\prime \prime}, \ldots$, so that $\operatorname{tcard}\left(C l_{z^{i}}(x), t\right)$ may vary. Then, we have a precise sense in saying that the quasi-cardinal of some qset whose elements are varying in number vary in time. The details of course can be easily fulfilled.

\section{Quantum Statistics within $\mathfrak{Q}$}

The derivation of quantum statistics depends on the assumption of the Indistinguishability Postulate (IP), which roughly says that permutations of indistinguishable quantum objects are not regarded as observable. In this section we will see that in using the language of quasi-set theory, we may avoid such an hypothesis. This of course has a price, namely, the complication of the language, since we need to work within the theory $\mathfrak{Q}$. But the gain in other places is interesting, for we can see, for instance, that in considering (truly) indistinguishable objects, we arrive 'naturally' to quantum statistics, what reinforces the idea that IP is imposed by the necessity of discourse, that is, its existence is due to the difficulty we have of dealing with $a b$ ovo indistinguishable objects within 'standard' languages as those of classical logic and mathematics. ${ }^{67}$ Let us provide a sketch of the main ideas involved in the derivation of the quantum distribution functions within $\mathfrak{Q}$; we begin by showing how to obtain the electronic distribution of an atom, for instance, in taking a sodium atom, we shall give a meaning, in terms of quasi-sets, to the expression $1 s^{2} 2 s^{2} 2 p^{6} 3 s^{1}$. Restricted quantifiers shall be used: so $\forall_{A} x \alpha$ means $\forall x(x \in A \rightarrow \alpha)$, while $\exists_{A} x \alpha$ stands for $\exists x(x \in A \wedge \alpha)$.

Suppose that $\mathcal{P}$ is a collection of non-empty pure qsets, that is, their elements are $m$-atoms. The elements of $\bigcup \mathcal{P}$ will be called quantum objects. ${ }^{68}$ Let $S$ be a finite and totally ordered 'set' (in the sense of $\mathfrak{Q}$ ) of quantum states, whose elements are denoted by $s_{1}, \ldots, s_{n}$. Furthermore, let $F$ be an unary predicate defined as follows: $F(x)={ }_{\text {df }} m(x) \wedge \forall_{S} s\left(x \in s \rightarrow q c(s)={ }_{E} 1\right)$. In words, $F(x)$ says that $x$ is a quantum object which cannot belong to a state with more than

\footnotetext{
${ }^{67}$ This section is based on Krause, Sant'Anna and Volkov 1999 an on Sant'Anna and Santos 2000.

${ }^{68}$ We remark that such an hypothesis has a physical motivation. For instance, we could be occupied with certain collections of particles, as those listed in a particle table. Furthermore, the elements of the collections in $\bigcup \mathcal{P}$ are not necessarily indistinguishable.
} 
one element; such an $x$ shall be called a fermion; furthermore, let us define $B(x)={ }_{\text {df }} m(x) \wedge \neg F(x)$ and call such an $x$ a boson. ${ }^{69}$

Now let $R$ be the quasi-relation

$$
R={ }_{\mathrm{df}}[[p, s]: p \in \mathcal{P} \wedge s \in S],
$$

which is subjected to the following restriction: for every $[p, s] \in R$, if $x \in p$ obeys $F(x)$, then $q c(p)={ }_{E} 1$. That is, there can be no more than one fermion in each quantum state. Call this restriction Pauli's Principle (PP).

For instance, let us consider our example of the electrons of a sodium atom. So, $q c(\bigcup \mathcal{P})=_{E} 11$ (there are eleven electrons to be distributed), and let $S={ }_{E}$ $\left\{s_{1}, \ldots, s_{12}\right\}$ be the set of possible states (the reason we are taking twelve states shall be made clear below). So, in this case the particular relation $R$ given by equation (7.1) is

$$
R={ }_{\mathrm{df}}\left[\left[p_{1}, s_{1}\right], \ldots,\left[p_{12}, s_{12}\right]\right],
$$

where $q c\left(p_{i}\right)={ }_{E} 1\left(i==_{E} 1, \ldots, 11\right)$, and $q c\left(p_{12}\right)={ }_{E} 0$.

In the general case, being defined the relation $R$ (equation (7.1), we can select a family $\left\{S_{i}\right\}_{i \in I}\left(I={ }_{E}\{1, \ldots, n\}\right)$ of subsets of $S$ such that $\bigcap S_{i}={ }_{E} \emptyset$. For instance, in our example, we may have

$$
\left\{S_{i}\right\}_{i \in\{1, \ldots, 12\}}==_{\mathrm{df}}\left\{\left\{s_{1}, s_{2}\right\},\left\{s_{3}, s_{4}\right\},\left\{s_{5}, s_{6}, s_{7}, s_{8}, s_{9}, s_{10}\right\},\left\{s_{11}, s_{12}\right\}\right\} .
$$

Each $S_{i}$ may be called an energy bin (or macrostate), while each element $s \in S_{i}$ may be called an energy state (or microstate). The mention of a particular $S_{i}$ (which is generally done by physical criteria) particularizes the collections of particles to certain interesting states, namely, the states that belong to the selected set $S_{i}$.

Taking our example once again, we see that each element of $\left\{S_{i}\right\}_{i \in\{1, \ldots, 12\}}$ (equation 7.3) corresponds to an energy level in the sodium atom. So, the element

$$
\left\{s_{5}, s_{6}, s_{7}, s_{8}, s_{9}, s_{10}\right\} \in\left\{S_{i}\right\}_{i \in\{1, \ldots, 12\}}
$$

corresponds to the energy level $2 p$, which encompasses 6 electrons, since each $s_{i}, i=5, \ldots, 10$ is associated to a quasi-set with just 1 quantum object (such a qset, remember, is a strong singleton). The element $\left\{s_{11}, s_{12}\right\}$ corresponds to the energy level $3 s$, which allows two electrons but in this case 'has' just one electron (recall that $q c\left(s_{11}\right)={ }_{E} 1$ and $q c\left(s_{12}\right)={ }_{E} 0$ ). The other two elements of $\left\{S_{i}\right\}_{i \in\{1, \ldots, 12\}}$, namely, $\left\{s_{1}, s_{2}\right\}$ and $\left\{s_{3}, s_{4}\right\}$, correspond respectively to the energy levels $1 s$ and $2 s$. The above equations induce the definition of the quasirelation

\footnotetext{
${ }^{69} \mathrm{So}$, if $\mathfrak{Q}$ is consistent, then no $x$ can be at the same time a fermion and a boson. The given definitions make the particles to be either a fermion or a boson, but we could modify them conveniently in order to admit within our description other kinds of particles as well, hence other kinds of statistics, as parastatistics, say -see French \& Krause's book. But in this section we shall be restricted to consider just bosons and fermions to exemplify the technique of using quasi-sets.
} 


$$
\begin{array}{r}
\bar{R}={ }_{E}\left[\left[p_{1} \cup p_{2},\left\{s_{1}, s_{2}\right\}\right],\left[p_{3} \cup p_{4},\left\{s_{3}, s_{4}\right\}\right],\right. \\
\left.\left[p_{5} \cup \ldots \cup p_{10},\left\{s_{5}, \ldots, s_{10}\right\}\right]\left[p_{11} \cup p_{12},\left\{s_{11}, s_{12}\right\}\right]\right],
\end{array}
$$

where $q c\left(p_{1} \cup p_{2}\right)={ }_{E} q c\left(p_{3} \cup p_{4}\right)={ }_{E} 2, q c\left(p_{5} \cup \ldots \cup p_{10}\right)={ }_{E} 6$ and $q c\left(p_{11} \cup\right.$ $\left.p_{12}\right)={ }_{E} 1$. This quasi-relation $\bar{R}$ is the quasi-set theoretical version for the usual expression $1 s^{2} 2 s^{2} 2 p^{6} 3 s^{1}$.

Now let us turn to the quantum statistics. Just to see the differences between the approach by using quasi-sets and the 'classical' one, we shall take another example. Informally speaking, suppose that we have two indistinguishable particles, labelled \#1 and \#2, distributed among two distinct states, specified by orthogonal wave-functions $\psi_{\mathbf{1}}$ and $\psi_{\mathbf{2}} \cdot{ }^{70}$ Then, due to the indistinguishability of the particles, we know that the corresponding vectors for bosons are

$$
\left|\psi_{\mathbf{1}}^{1}\right\rangle \otimes\left|\psi_{\mathbf{2}}^{1}\right\rangle,\left|\psi_{\mathbf{2}}^{1}\right\rangle \otimes\left|\psi_{\mathbf{1}}^{1}\right\rangle \text { and } \frac{1}{\sqrt{2}}\left(\left|\psi_{\mathbf{1}}^{1}\right\rangle \otimes\left|\psi_{\mathbf{2}}^{2}\right\rangle+\left|\psi_{\mathbf{2}}^{1}\right\rangle \otimes\left|\psi_{\mathbf{1}}^{2}\right\rangle\right)
$$

while, in the case of fermions, we have

$$
\frac{1}{\sqrt{2}}\left(\left|\psi_{\mathbf{1}}^{1}\right\rangle \otimes\left|\psi_{\mathbf{2}}^{2}\right\rangle-\left|\psi_{\mathbf{2}}^{1}\right\rangle \otimes\left|\psi_{\mathbf{1}}^{2}\right\rangle\right)
$$

So, the labels \#1 and \#2, originally attached to the particles, were veiled by the adequate choose of the vectors which express symmetry conditions, as in the usual approach. In order to see how quasi-sets enter in the discussion, let us suppose now that we have defined a quasi-relation $R$ as in equation (7.1), that is, $R={ }_{E}[[p, s]: p \in \mathcal{P} \wedge s \in S]$. In other words, we are considering a certain collection of elementary particles (say, particles of several kinds), and let us suppose that $q c(\bigcup \mathcal{P})=_{E} \nu_{i}$ (this is the total number of particles), subjected to certain states which are taken as elements of a collection $S$ of states. Then, again as above, suppose that we are able to select a family $\left\{S_{i}\right\}_{i \in I}$ of subsets of $S$ such that $\bigcap_{i \in I} S_{i}={ }_{E} \emptyset$, where $I={ }_{E}\left\{1,2,3, \ldots, k_{i}\right\}$. Then, for any particular situation $i$, we can define the quasi-relation $\left.R\right|_{i}={ }_{E}\left[[p, s]: p \in \mathcal{P} \wedge s \in S_{i}\right]$.

Each relation $\left.R\right|_{i}$ describes intuitively a particular distribution of the $\nu_{i}$ particles in the $k_{i}$ states of $S_{i}$. So, if we intend to answer the fundamental question: 'In how many ways can we correspond (or distribute) $\nu_{i}$ indistinguishable bosons in $k_{i}$ quantum states ?', since a correspondence between bosons and quantum states is given by the quasi-relations $\left.R\right|_{i}$, and taking into account that we are talking about bosons, which are not subject to the restriction imposed by the Pauli Principle mentioned above, the answer is precisely the quantity of quasirelations $\left.R\right|_{i}$ that can be performed.

Let us explain this last assertion by considering a particular case. Suppose that $\nu_{i}={ }_{E} 5$; so, we have five indistinguishable bosons to be distributed among,

\footnotetext{
${ }^{70}$ Sant'Anna and Santos 2000 have considered also a number $k>2$ of particles and a number $n>2$ of states.
} 
say, 3 distinct cells (states) $s_{1}, s_{2}$ and $s_{3}$. That is, $q c\left(S_{i}\right)={ }_{E} 3$. Since collections of bosons with the same quasi-cardinality are indistinguishable in the sense of the Weak Extensionality Axiom, we shall refer to them by their quasi-cardinalities only. Thus, there are 21 different ways of distributing 5 indistinguishable bosons into states $s_{1}, s_{2}$ and $s_{3}$, that is, there are 21 possible quasi-relations $\left.R\right|_{i} ^{j}$, $j={ }_{E} 1, \ldots, 21$ that can be defined for this particular situation $i$. In the present example, these quasi-relations are shown in the table below, where the number of entries stand for the quasi-cardinality of the quasi-sets $p_{1}, p_{2}$ and $p_{3}$ associated to each $s_{i}$; the numbers $1, \ldots, 21$ name the relations $\left.R\right|_{i} ^{1}$ to $\left.R\right|_{i} ^{21}$ :

\begin{tabular}{|l|l|l|l|l|l|l|l|l|l|l|l|l|l|l|l|l|l|l|l|l|l|}
\hline & 1 & 2 & $\mathbf{3}$ & 4 & 5 & 6 & 7 & 8 & 9 & 10 & 11 & 12 & 13 & 14 & 15 & 16 & 17 & 18 & 19 & 20 & 21 \\
\hline$s_{1}$ & 5 & 4 & $\mathbf{4}$ & 3 & 3 & 3 & 2 & 2 & 2 & 2 & 1 & 1 & 1 & 1 & 1 & 0 & 0 & 0 & 0 & 0 & 0 \\
\hline$s_{2}$ & 0 & 1 & $\mathbf{0}$ & 0 & 1 & 2 & 0 & 1 & 2 & 3 & 0 & 1 & 2 & 3 & 4 & 0 & 1 & 2 & 3 & 4 & 5 \\
\hline$s_{3}$ & 0 & 0 & $\mathbf{1}$ & 2 & 1 & 0 & 3 & 2 & 1 & 0 & 4 & 3 & 2 & 1 & 0 & 5 & 4 & 3 & 2 & 1 & 0 \\
\hline
\end{tabular}

For instance, in the third emphasised column, we have the quasi-relation

$$
\left.R\right|_{i} ^{3}={ }_{E}\left[\left[p_{1}, s_{1}\right],\left[p_{2}, s_{2}\right],\left[p_{3}, s_{3}\right]\right],
$$

where $q c\left(p_{1}\right)={ }_{E} 4, q c\left(p_{2}\right)=0$ and $q c\left(p_{3}\right)={ }_{E} 1$.

This table shows that the number of ways we have to distribute $\nu_{i}$ indistinguishable bosons in the $k_{i}$ quantum states $s$ is 21 , a number which is usually obtained by Einstein's equation:

$$
I_{\text {bosons }}^{i}={ }_{E} \frac{\left(k_{i}+\nu_{i}-1\right) !}{\left(k_{i}-1\right) ! \nu_{i} !} .
$$

So, Einstein's equation expresses here the way of calculating the number of possible relations $\left.R\right|_{i} ^{j}$ that can be performed in each situation $i .{ }^{71}$ In the same way, if we repeat our calculations for fermions; due to the Pauli Principle, we have:

$$
I_{\text {fermions }}^{i}={ }_{E} \frac{k_{i} !}{\left(k_{i}-\nu_{i}\right) ! \nu_{i} !} .
$$

An important remark is that usually these last equations are obtained by considering that different quantum states correspond to distinct energy levels. Our calculations are more general in the sense that we may have different quantum states at the same energy level as in the case of the sodium atom considered above. In interpreting quantum states as energy levels, we could consider, for example, that $S$ should be an energy interval given by $[0, k T]$, where $k$ is the Boltzmann constant and $T$ is, say, $300 K$, while each $S_{i}$ corresponds to an energy range of about $10^{-33} \mathrm{~J}$ (there are $10^{12} S_{i}$ 's), and each $S_{i}$ has $10^{19}$ quantum states $s$.

\footnotetext{
${ }^{71}$ Recall that the numbers involved in the formula are quasi-cardinals of certain qsets.
} 
The total number of microstates corresponding to a given macrostate (energy bin) is given by

$$
I_{\text {bosons(fermions) }}={ }_{E} \prod_{i} I_{\text {bosons(fermions) }}^{i} .
$$

Since classical mathematics can be obtained within the scope of quasi-set theory, all the calculations that follow the derivation of the statistics can be performed here as usual. The most probable macrostate will be determined by maximizing $\log I-\alpha N-\beta E$, where $\alpha$ and $\beta$ are Lagrangian parameters that have been introduced to take into account the restriction of fixing the total particle number $N$, total energy $E$, and $\log$ is the natural logarithm. Thus, it seems clear that we need to define an injective q-function $f: S \rightarrow \mathcal{E}$, where $\mathcal{E}$ is an interval of positive real numbers. Intuitively, $\mathcal{E}$ corresponds to energy.

In the case of fermions we should maximize the following q-function $F$ :

$$
\begin{array}{r}
F={ }_{\mathrm{df}} \log \left(\prod_{i} \frac{k_{i} !}{\left(k_{i}-\nu_{i}\right) ! \nu_{i} !}\right)-\alpha \sum_{i} \nu_{i}-\beta \sum_{i} \nu_{i} \varepsilon_{i}= \\
\sum_{i}\left[k_{i}\left(\log k_{i}-1\right)-\left(k_{i}-\nu_{i}\right)\left(\log \left(k_{i}-\nu_{i}\right)-1\right)\right. \\
\left.-\nu_{i}\left(\log \nu_{i}-1\right)-\alpha \nu_{i}-\beta \varepsilon_{i} \nu_{i}\right],
\end{array}
$$

where $\varepsilon_{i}$ stands for the energy associated for each $S_{i}$. It is clear from these calculations that we have used Stirling's approximation, which states that $\log K ! \approx$ $K(\log K-1)$ for $K \gg 1$. Nevertheless, such an approximation was used just for bin occupation numbers $\nu_{i}$ and not for the state occupation numbers.

If

$$
\frac{\partial F}{\partial \nu_{i}}={ }_{E} 0
$$

there results that

$$
\nu_{i}={ }_{E} \frac{k_{i}}{e^{\alpha+\beta \varepsilon_{i}}+1} .
$$

If we assume that the energy differences of the states in the $i$-th bin are negligible, then according to equation (7.13), the average occupation of any individual state in that bin is

$$
\frac{\nu_{i}}{k_{i}}={ }_{E} \frac{1}{e^{\alpha+\beta \varepsilon_{i}}+1}
$$

Finally, the average occupation number of the $n$-th single-particle state of energy $\varepsilon_{n}$ is given by the well-known Fermi-Dirac distribution function:

$$
f_{\text {fermions }}={ }_{E} \frac{1}{e^{\alpha+\beta \varepsilon_{n}}+1} .
$$


For bosons, the calculations are very similar, and we have the Bose-Einstein distribution function:

$$
f_{\text {bosons }}={ }_{E} \frac{1}{e^{\alpha+\beta \varepsilon_{n}}-1} .
$$

The physical interpretation of the parameters $\alpha$ and $\beta$ is standard: $\beta={ }_{E}$ $1 / k T$, where $k$ is Boltzmann constant and $T$ is the absolute temperature, while $\alpha$ is a normalization constant usually referred to as affinity.

The helium atom is probably the simplest realistic situation where the problem of individuality plays an important role. With identity question put aside, the wave function of the helium atom would be just the product of two hydrogen atom wave functions with $Z={ }_{E} 2$ instead of $Z={ }_{E} 1$. Nevertheless, the space part of the wave function for the case where one of the electrons is in the ground state (100) and the other one is in excited state $(\mathrm{nlm})$ is:

$$
\phi\left(\mathbf{x}_{\mathbf{1}}, \mathbf{x}_{\mathbf{2}}\right)={ }_{E} \frac{1}{\sqrt{2}}\left[\psi_{100}\left(\mathbf{x}_{\mathbf{1}}\right) \psi_{n l m}\left(\mathbf{x}_{\mathbf{2}}\right) \pm \psi_{100}\left(\mathbf{x}_{\mathbf{2}}\right) \psi_{n l m}\left(\mathbf{x}_{\mathbf{1}}\right)\right]
$$

where the $+(-)$ sign is for the spin singlet (triplet) ${ }^{72}$ and $\mathbf{x}_{\mathbf{1}}$ and $\mathbf{x}_{\mathbf{2}}$ are position vectors of the electrons.

For the ground state, however, the space function needs to be necessarily symmetric. In this case, the problems regarding identity have no physical effect. The most interesting case is certainly the excited state. Equation (7.17) reflects our ignorance on which electron is in position $\mathbf{x}_{\mathbf{1}}$ and which one is in position $\mathbf{x}_{\mathbf{2}}$. Nevertheless, in the same equation there are terms like $\psi_{\mathbf{1 0 0}}\left(\mathbf{x}_{\mathbf{1}}\right)$, which corresponds to a specific physical property of an individual electron.

Our quasi-set theoretical interpretation of equation (7.17) is the following (it resembles the case of the sodium atom discussed above). Let $P$ be a pure quasiset such that $q c(P)={ }_{E} 2$. We intuitively interpret the elements of $P$ as electrons of the Helium atom. If $G$ is a unary predicate such that $G(x)$ intuitively says that ' $x$ is in the ground state' (the definition of $G$ depends on physical aspects), then, by using the Separation Axiom of $\mathfrak{Q}$, we obtain the sub-quasi-set $p_{1} \subseteq P$ defined by

$$
p_{1}=_{\mathrm{df}}[x \in P: G(x)] .
$$

If we call $p_{2}={ }_{\mathrm{df}} P-p_{1}$, then $q c\left(p_{1}\right)={ }_{E} q c\left(p_{2}\right)={ }_{E} 1$. So, the elements of $p$, despite their indistinguishability, are 'separated' by their 'respective states'. More formally, by calling $g_{1}$ the ground state and $g_{2}$ the another state, we may define

$$
R={ }_{\mathrm{df}}\left[\left[p_{1}, g_{1}\right],\left[p_{2}, g_{2}\right]\right] .
$$

It is clear that $g_{1}$ and $g_{2}$ may be interpreted respectively as 100 and $\mathrm{nlm}$ as above. So, we have obtained a way of expressing that between two objects (the

\footnotetext{
${ }^{72}$ Spin singlet refers to total spin zero and spin triplet refers to total spin different of zero.
} 
elements of $P$ ) there is one of them in the ground state, although we cannot identify which one, since the qsets $p_{1}$ and $p_{2}$ are indistinguishable (in the sense of the Weak Extensionality Axiom). In other words, equation (7.19) stands for the situation presented in equation (7.17).

We still need to show how the quasi-function $R$ evolves in time, that is, we need to explain the sense according to which equation (7.19) plays the role of the wave function given in equation (7.17). This may be achieved if we consider the modified quasi-set theory $\mathfrak{Q}^{t}$ described above, in which the quasi-cardinals may vary in time. But this would complicate in much the mathematical details. ${ }^{73}$

\section{Conclusion: on justifying Quasi-Set Theory}

If the reader agrees with Hilbert that the mathematician (so as the philosopher) is free for investigating all possible theories, and not only those which are near to reality (Hilbert 1902), no justification for developing quasi-set theory in in need. But, if he/she is like F. Gonseth (Gonseth 1974) and thinks that logic (here understood in the sense of the 'great logic', that is, involving also set theory) is the physics of the object whatever, then it makes sense to ask for 'the logic' which underly certain empirical domains, in particular the quantum domain. It is well known that ever since the 1930s von Neumann (and, later, he and G. Birkhoff) proposed the directions of 'quantum logics', which turn to be more the algebraic study of some lattices that the study of the 'logic' which underly quantum mechanics itself. ${ }^{74}$

Believing in quantum mechanics, and following this second direction, we should regard the basic entities of the same kind as indiscernible, right at the start, as required by H. Post (1963), and so we should not promote some kind of ad hoc device for treating them as they were indiscernible, as what happens when some form of symmetrization postulate is introduced. Anyway, it is a pertinent philosophical question to look for the kind of 'logic' these indiscernible entities do obey. In such a logic, of course we would be able to talk of indiscernibility, and to consider that some entities may have all their relevant properties in common without turning to be the very same entity, as implied by Leibniz Law.

Two directions are open to us in this endeavor. The first is that one which 'makes physics work'. This is achieved by finding an adequate mathematical description of quantum theory in which the concept of indiscernibility can be dealt with. This route gives us the standard approaches to microphysics, and being developed within a standard mathematical framework, corresponds to restrict the theory to some mathematical structure built in set theory. But then it is necessary to introduce some kind of principle of symmetry, which (summing up) says that permutations of (supposed) indiscernible entities do not give a

\footnotetext{
${ }^{73}$ Sant'Anna and Krause have suggested a way of obtaining Schrödinger's equation in $\mathfrak{Q}$, but this will be not pursued here. Sant'Anna \& Krause 1998.

${ }^{74}$ For a general survey on quantum logics in this last sense, see Dalla Chiara \& Giuntini 2001.
} 
distinct situation than that one we had before the permutation. An example of this situation if the selection of symmetric and anti-symmetric vectors of the relevant Hilbert space as representing physical phenomena.

But we could be interested in following Heinz Post suggestion that the indiscernibility of quantum entities should be considered as their very peculiar characteristic, not as something made a posteriori by ad hoc devices, but as a primitive notion. This is what quasi-set theory aims to do. It enable us to consider quantum objects as really and truly indistinguishable entities $a b$ ovo. The price to be payed is a complication in the underlying language, as we have seem, for quasi-sets are less intuitive than standard sets. But there is at least one example in the scientific literature which may inspire us (preserved the difference of importance in both cases and without comparing them of course); as it is well known, Albert Einstein used a non-Euclidian geometry in his relativity theory. Such a 'complication' of the underlying mathematics had a role to play, according to Rudolf Carnap. Carnap tells us that the use of non-Euclidean geometry for space in relativity theory, despite the mathematical complications in relation to the well known Euclidean geometry, caused "an enormous simplification of physical laws" so avoiding the use of "weird laws". 75 The use of quasi-sets for the treatment of some concepts in quantum theory may be useful for similar purposes, at least in what respects philosophical aspects of the concept of non-individual quanta.

\section{Acknowledgment}

The content of this paper is part of Chapter 7 of S. French \& D. Krause's Identity and Individuality in Modern Physics, which is still in preparation, and corresponds to an extension of a previous work (not published yet) written jointly with Adonai Sant'Anna and Aurelio Sartorelli (Sartorelli et al. 2001). The philosophical discussions involving non-individuality are developed in the book just mentioned and is not explored here. I would like to thank several people with whom I have discussed quasi-sets, specially Adonai Sant'Anna and Aurelio Sartorelli, so as Antonio Coelho, Maria Luisa Dalla Chiara, Newton da Costa, Steven French, although all the faults the reader will find here are of course mine. A version of this work appeared as a pre-print at the Institute for Advanced Studies (IEA) of the University of São Paulo, in the series Coleção Documentos of the IEA/USP, No. 46, March 2003.

\section{References}

[1] Bitbol, M.: 1996, Mcanique Quantique: une Introduction Philosophique, Paris, Flammation.

[2] Cantor, G.: 1955, Contributions to the Founding of the Theory of Transfinite Numbers, New York, Dover.

${ }^{75}$ Carnap 1995, p. 164. 
[3] Carnap, R.: 1995, An introduction to the philosophy of science, New Yoir, Dover (ed. Martin Gardner).

[4] Cocchiarella, N. B.: 1995, 'Review of 'Individuals, kinds and names in physics', Mathematical Reviews, 95a:03018.

[5] da Costa, N. C. A.: 1980: Ensaio sobre os fundamentos da lógica, São Paulo, Hucitec/EdUSP (2nd. ed. Hucitec 1994).

[6] da Costa, N. C. A. and Krause, D.: 1994, 'Schrödinger logics', Studia Logica 53 (4), 1994, 533-550.

[7] da Costa, N. C. A. and Krause, D.: 1997, 'An intensional Schrödinger logic', Notre Dame Journal of Formal Logic 38 (2), 1997, 179-194.

[8] da Costa, N. C. A. and Krause, D.: 1999, 'Set-theoretical models for quantum systems', in Dalla Chiara, M. L. et. al. (eds.), Language, quantum, music, Kluwer Ac. Pu., pp. 171-181.

[9] Dalla Chiara, M. L.: 1987, 'An approach to intensional senmantics', Synthese 73, 1987, pp. 479-496.

[10] Dalla Chiara, M. L. and Toraldo di Francia, G.: 1993, 'Individuals, kinds and names in physics', in Corsi, G. et al. (eds.), Bridging the gap: philosophy, mathematics, physics, Kluwer Ac. Pu., 261-283.

[11] Dalla Chiara, M. L. and Toraldo di Francia, G.: 1995, 'Identity Questions from Quantum Theory", in Gavroglu, K. et. al. (eds.), Physics, Philosophy and the Scientific Community, Dordrecht, Kluwer Academic Publishers, pp. 39-46

[12] Dalla Chiara, M. L., Giuntini, R. and Krause, D.: 1998, 'Quasiset theories for microobjects: a comparision', in Castellani, E. (ed.), Interpreting Bodies: Classical and Quantum Objects in Modern Physics, Princeton, Princeton Un. Press, pp. 142-152.

[13] Dalla Chiara, M. L. and Giuntini, R.: 2001, 'Quantum logic', in http://xxx.lanl.gov/list/quant-ph/0101

[14] Enderton, H. B.: 1977, Elements of set theory, Academic Press.

[15] French, S. and Rickles, D. P.: 2002, 'Understanding permutation symmetry', pre-print, University of Leeds.

[16] French, S. and Krause, D.: 2002, Identity and Individuality in Modern Physics, forthcoming.

[17] Gonseth, F.: 1974, Les mathmatiques et la ralit: essay sur la mthode axiomatique, Albert Blanchard, 1974 (original from 1936). 
[18] Hatcher, W. S.: 1982, The logical foundations of mathematics, Pergamon Press.

[19] Kawking, S.: 2001, O Universo numa casca de noz, Mandarim.

[20] Hilbert, D.: 1902, 'Mathematical problems', Bull. American Mathematical Society 8, 437-479, translated by M. W. Nelson from 'Mathematische probleme', Archiv der Math. u. Phys. 1, 1901, 44-63 and 213-237. Reprinted in Browder 1976, pp. 1-34.

[21] Hume, D., Treatise of human nature, 2nd. ed. L. A. Selby-Bigge, Oxford, Oxford Un. Press, 1985.

[22] Ignatieff, Yu. I.: 1996, The mathematical world of Walter Noll, Springer.

[23] Krause, D.: 1990, Não-Refexividade, Indistinguibilidade e Agregados de Weyl, Tese de Doutoramento, FFLCH (Filosofia), Universidade de São Paulo.

[24] Krause, D.: 1991, 'Multisets, quasi-sets and Weyl's aggregates', J. NonClassical Logic 8 (2), pp. 7-23.

[25] Krause, D.: 1992, 'On a quasi-set theory', Notre Dame J. of Formal Logic 33, pp. 402-411.

[26] Krause, D.: 1996, 'Axioms for collections of indistinguishable objects', Logique et Analyse 153-154, pp. 69-93.

[27] D. Krause, A. S. Sant'Anna and A. G. Volkov: 1999, 'Quasi-set theory for bosons and fermions: quantum distributions', Foundations of Physics Letters 12 (1), pp. 51-66.

[28] Krause, D. and French, S.: 1995, 'A Formal Framework for Quantum Non-Individuality', Synthese 102, 195-214.

[29] Krause, D. and French, S.: 1999, 'Opaque predicates, veiled sets and their logic', in Carnielli, W. A. and D'Ottaviano, I. M. L. (eds.), Advances in Contemporary Logic and Computer Science, Proceedings of the Eleventh Brazilian Conference on Mathematical Logic (Salvador, Bahia, May 610, 1996), American Mathematical Society (Contempory Mathematics, Vol. 235), pp. 263-274.

[30] Krause, D. and Coelho, A. M. N.: 2002, 'Identity, indiscernibility and philosophical claims', CLE e-prints, Center for Logic, Epistemology and the History of Science, Un. of Campinas, Vol. 2, No. 3 (February). Forthcoming in Axiomathes.

[31] Manin, Yu. I.: 1976, 'Mathematical Problems I: Foundations', in Browder, F. E. (ed.): 1976, Mathematical Problems Arising from Hilbert Problems, Proceedings of Symposia in Pure Mathematics, Vol. XXVIII, Providence, American Mathematical Society, p. 36. 
[32] Mendelson, E.: 1997, Introducttion to Mathematical Logic, Chapmann \& Hall, 4th. ed.

[33] Penrose, R.: 1989, The emperor's new mind, Oxford Un. Press.

[34] Post, H.: 1963, The Listener, 10 October, pp. 534-537, reprinted in Vedanta for East and West 132, 1973, pp. 14-22.

[35] Sant'Anna, A. S. and Santos, A. M. S.: 2000, 'Quasi-set theoretical foundations of statistical mechanics: a research program', Foundations of Physics 30 (1), pp. 101-120.

[36] Sartorelli, A., Krause, D. and Sant'Anna, A. S.: 2001, 'A critical study on the concept of identity in Zermelo-Fraenkel-like axioms', xxx.lanl.gov/list/math.LO/0106098 (12 June 2001).

[37] Schrödinger, E.: 1952, Science and Humanism, Cambridge, Cambridge Un. Press.

[38] Shoenfield, J. R., Mathematical Logic, Addison Wesley, 1967.

[39] Simons, P.: 1987, Parts: a study in ontology, Oxford, Clarendon Press.

[40] Skolem, T.: 1922, 'Some remarks on axiomatized set theory', in van Heijenoort, J. (ed.), From Frege to Gödel: a source book in mathematical logic 1879-1931, Harvard Un. Press, 1967, pp. 290-301.

[41] Suppes, P.: 1957, Introduction to logic, Van Nostrand.

[42] Suppes, P.: 1960, Axiomatic Set Theory, Dover Pu. 1972 (first editon by van Nostrand 1960).

[43] Suppes, P.: 1967, Set theoretical structures in science, mimographed notes, Stanford Un.

[44] Suppes, P.: 2002, Representation and Invariance of Scientific Structures, CSLIPu. Chicago Un. Press.

[45] Toraldo di Francia, G.: 1978, 'What is a physical object?', Scientia 113, pp. 57-65.

[46] Toraldo di Francia, G.: 1990, Un universo troppo simplice: la visione historica e la visione scientifica del mondo, Feltrinelli, Milano. 